\title{
Why is the term 'developmental bias' misleading? (full version)
}

\author{
Andrzej Gecow \\ andrzejgecow@gmail.com
}

\begin{abstract}
The term 'developmental bias' promoted in the Special Issue of 'Evolution \& Development' was initially defined as the effect in evolution direction creating by correlation of changed developmental parameters. The understanding of the term is in the Special Issue much broader and very poorly defined. Promoting such a broad term related to observation makes difficult to explain specific mechanisms. It is now time for the task of explaining to become more important in biology than just collecting facts. The term requires quick organization and division into many different concepts. I suggest replacing this term with a shorter one (devbias) with an additional tip indicating a specific variant according to the proposed ordering system. Mainly the mechanisms giving variability clearly focused on greater fitness (devbiases4) have been added. Devbias4 was treated in Special Issue as an independent source of adaptation supporting natural selection. Focusing on the time interval of current evolution, where collected devbiases4 indeed supports natural selection, it is usually forgotten to stress that they are also the result of previous natural selection and only mediate the transmission of its effects. This leads to a dangerous message beyond biology that scientific observations have shown that the Darwinian mechanism is not the only source of adaptation. By the way of ordering 'devbias' the understanding of 'randomness' is discussed to show differences between 'flat distribution', 'variation blind on needs' and 'real random variability' which in Special Issue are typically mixed.
\end{abstract}

Keywords developmental bias; plasticity; adaptation source; Lamarckian mechanisms.

\section{Introduction}

Special Issue of 'Evolution \& Development' titled 'Developmental Bias in Evolution' edited by Armin Moczek was published in January 2020. In EDITORIAL chapter titled 'Biases in the study of developmental bias' Moczek writes: "In November 2018 the Santa Fe Institute hosted a two-day workshop titled Developmental Bias and Evolution, funded by a grant from the John Templeton Foundation. Involving 34 participants and 22 talks, ... This special issue is meant to capture this diversity of viewpoints, and to provide a collection of perspectives that will inform and motivate the next round of research, and the next generation of researchers." So, the main aim of this Issue is promotion of the term 'developmental bias' as symbol of scientific problem ${ }^{1}$. I feel motivated to respond to this invitation.

I met this Special Issue looking for current explanation of sources of adaptations in evolution via plasticity and other mechanisms called follow (Jablonka at al. 1998, 1995) 'Lamarckian mechanisms'. This topic is unlucky to name, both 'developmental bias' and 'Lamarckian mechanisms' strongly suggest a misunderstanding of such terms. I have just discussed this theme to relax this misunderstanding and tried to publish (Gecow 2019a) ${ }^{2}$. Indeed, this Special Issue gave me many news in the topic. It seems like another, independent world than publications of Jablonka, who is there not noted at all. The same phenomena are there described in different notions and in different philosophy of methodology. Why? - it is a question.

I afraid, I have an answer. As probably is suggested ${ }^{3}$ in (Pocheville, Danchin 2016) Jablonka is treated as a neo-Lamarckian, probably only because she openly used term 'Lamarckian dimension of evolution' (Jablonka at al. 1995, 1998, 2005), and as such she is carefully omitted by authors, to be not included to this set of 'outmoded scientists'. It is too shallow motivation, but I afraid, it works. Jablonka does not consider other phenomena than evo-devo does in Special Issue, but she looks for particular, causal mechanisms, therefore she uses other terms. Current definition of neo-Lamarckism found in $\mathrm{Wiki}^{4}$ (it is wide and emotional range of such definitions) includes 'development-first' approach of West-Eberhard (2003) and 'plasticity-led evolution (i.e., "PLE”)' (Levis, Pfennig

\footnotetext{
1 "Less crucial is a definition ... Instead, what we should want is a concept that generates intellectual identity across various researchers, and that unites the diverse fields and approaches relevant to the study of developmental bias, from paleontology to behavioral biology." (Brigandt 2020).

${ }^{2}$ Currently this article is much upgraded what will be available soon.

3 "The question of blind variation is probably the most salient stumbling block of the old and reviving debates between neo-Darwinians and neo-Lamarckians [e.g. Romanes, 1888; Jablonka and Lamb, 1995; 2010].” (Pocheville, Danchin 2016).

4 ...theories involving the inheritance of characteristics acquired during an organism's lifetime. Scientists who felt that such Lamarckian mechanisms were the key to evolution were called neo-Lamarckians."

[https://en.wikipedia.org/wiki/Lamarckism\#Neo-Lamarckism July 27, 2019].
} 
2020), but nobody afraid promoting them, that he will be taken as neo-Lamarckian. The source of adaptations is much more important difference connected to dichotomy Lamarck's and Darwin's approaches. Darwin shows stochastic, natural selection mechanism but Lamarck suggested, that causal 'mechanical' mechanisms exist. The view similar to Lamarck's is suggested by many of authors of Special Issue and to clarify this misunderstanding I write this article. Let us resign classifying what is Lamarckian or neo-Lamarckian. For such classification firstly we should agree, how look like Lamarck's view, but it is no place for such debate in this article. Currently this theme is too wide and containing too many distortions and misjudgements, and too emotional. I try to explain the basic misunderstandings in (Gecow 2019a).

Reading carefully all articles from Special Issue, I can agree, that sources of adaptations are there (typically) correctly indicated, however, first look gives another, surprising conclusion. It may be not intended by the authors. It implies from used methodology which implies from a philosophy. Biologists do not think about these foundations, however they should. They apply the tradition uncritically. Sorry. I consider this methodology as too old and currently impeding explanation and theoretical summery. In effect, the same applies to the term 'developmental bias' which has to be a symbol of this approach and therefore its promotion is the main aim of this issue. The first look is different than later conclusion, but it is especially important, due it will be peck upped by not-scientists and announced as scientific conclusion in the socially very hot and even politically sensitive ideological controversy. It can be easily expected, that in effect the evolutionary science will not gain but lose. Therefore the first aim of this article is to neutralize such misleading 'scientific conclusions'. The main of them is that there are other mechanisms leading to the adaptation than natural selection. I will state, that indicated as such mechanisms some developmental biases are only an intermediary between earlier works of natural selection and current use of previously prepared results.

The traditional biological methodology leading to introduce the term 'developmental bias' prefers the most detailed description of phenomena and treats explanation as less scientific job, especially abstract 'speculation' as not worthy ${ }^{5}$ of biologists. Such old and extreme approach is probably the result of disappointment and increased caution when explanations of e.g. recapitulation based on inheritance of acquired characters proved to be incorrect. The term 'developmental bias' has to describe observed phenomena without any suggestion to particular explanation. In effect it is very poorly defined (what agree most of authors ${ }^{6}$ ) and contains too wide range of different phenomena, to be useful for next step - mechanisms identification necessary for explanation.

I am not saying that the term 'developmental bias' is useless. It is obvious that to get to the explanation stage, we must first describe the hypothetical phenomenon on as many examples as possible. The articles (Jablonski 2020; Jackson 2020; Hu et al. 2020) and others from this collection are examples of this way. However, replacing 'plasticity' with a supposedly more general 'developmental bias' is a step back to the less defined term, that requires greater variety of explanatory mechanisms. This direction is not a generalization by weakening the assumptions in a particular mechanism, which allows it to be used to explain a larger set of facts. It is a completely different generalization. I have the same complaint about the tendency to such extension of the term 'plasticity' On the current stage the collected phenomena should be at the beginning selected to separate boxes defined much more exactly than 'development bias' is (in practices). This is just available, but for this the stress must be shifted from description of an effect to indication of a mechanism. Therefore, the second aim of my article is to define such set of the boxes to allow a segregation of the capacity of bag 'developmental bias'. For this I will start from analysis of initial, Arthur's (2004) definition and later, definition used in (Uller et al. 2018) which is practically the main used in the Special Issue. I expect, I will not found all needed boxes, but I hope that this initial order will make it easier to navigate the topic.

\footnotetext{
${ }^{5}$ However, in (Levis, Pfennig 2020) term 'speculation' is used in correct and positive sense: "Here, we begin to address these shortcomings by first speculating about how various features of development - modularity, flexible regulation, and exploratory mechanisms - might impact and/or bias whether and how PLE unfolds." 6 (Brigandt 2020): "the term "developmental bias" has scarcely been used. And one may even doubt whether a unique and principled definition of bias is possible."

(Laland et al. 2020) "The term "developmental bias," as commonly deployed, is somewhat ambiguous because it is manifestly subject to two distinct readings ... One way of resolving the ambiguity is to distinguish between the two usages explicitly: that is, distinguishing between a biased product of development (henceforth „developmentally biased phenotypic variation”), and a biasing process of development ( „developmentally biased evolutionary process")."

${ }^{7}$ See newer version of (Gecow 2019a) if it is already available.
} 


\section{Theoretical approach initiated by Arthur's definition}

\subsection{Arthur's definition of 'developmental bias'}

The basic suggestion of the meaning the term 'developmental bias' promoted in Special Issue is the same as has occur earlier in (Uller et al. 2018), where 'developmental bias' is defined as "some variants [of phenotype] arising more readily than others". This definition refers to 'possible phenotype space', is similar wording to its prototype, but much wider understood.

It is based on earlier ${ }^{8}$ work of Arthur (2004) who describes it as follow: "Let us suppose that there are n possible directions in which the ontogenetic trajectory can be rerouted. If, summed over a very large number of different mutations of the gene concerned, the probability of being rerouted in some directions is higher than for other directions, then there is developmental bias. Alternatively, if the probability is the same for all $\mathrm{n}$ directions, then there is no bias." or "For two characters that can be measured in the same units, ... bias can be said to exist when the pattern of covariation takes any shape other than a circle." He shows using clear figures, how he understands new term 'developmental bias'.

For me, the message of the article (Arthur 2004) is clear: Arthur's intention is to introduce a more complete concept containing in addition to 'developmental constraints' also 'developmental drive'. Thus, he presents a theoretical model demonstrating the unity of this mechanism. In this model, a key role is played by the correlation between changes in various phenotypic features in the distribution of probabilities of changes proposed for selection, i.e. in the distribution of changes before selection. So it does not depend on the selection or distribution of fitness (Wright's landscape), which will work later. As an independent factor together with the fitness landscape during selection directs evolution. Because this factor is already determined before selection, Arthur calls it 'bias'.

Because of the above intention, Arthur is not concerned with the possibility of targeting changes within one feature in the distribution prior to selection, so he does not mention the lack of such targeting, but he tacitly accepts such an assumption. In such a case, he may state that if the distribution does not show such correlation, then the direction of evolutionary variability after selection does not depend on this distribution - there are no bias. However, if these correlations occur, they usually affect the direction of evolution. Bias defined in this way applies only to this one theoretical correlation-based mechanism.

We can treat the mechanism shown as an example, and the name as open to similar mechanisms, if found. The suggested essence of this name was that the factor present in the distribution of phenotypic variation before selection, independent of the fitness distribution active in the later selection, influences the direction of evolution. There is no suggestion that this factor has to increase the average fitness of the proposed changes.

For convenience, a specific, current fitness landscape will be included in the term '(natural) selection'.

Arthur, however, had a serious problem with indicating in (Fig. 1 Arthur 2004) such an experimental distribution (rather such was expected by the recipients), because it is only theoretical distribution, one can say virtual, rather impossible to measure, which does not mean that does not exist and is not relevant for explanatory considerations. It is about the distribution of changes in phenotypic traits, because selection sees them. Even if the distribution of possible genetic changes could be determined, this initial point can hardly be called a phenotypic developmental stage. At each subsequent stage, selection has already worked.

To illustrate the impact of feature correlation on evolution, Arthur used in (Fig. 1 Arthur 2004) the distribution of 'population's phenotypic variation', indicating 'M, population mean'. It cannot be a distribution of the state of diversity of phenotypes in a population at a given moment, resulting from the current diversity of genotypes and the current set of alleles and their frequency, because it should be about possible changes, not about the state, and about changes before selection, but such a state is already after selection during development. However, this interpretation comes as first. By the way, it would be interesting to determine how similar these distributions are. So Arthur avoided determining whether it is a theoretical or experimental distribution and how it can be obtained.

To sum up, the bias defined by Arthur relates to the factor in the theoretical distribution of phenotypic changes before selection influencing the direction of evolution.

\subsection{Extension of Arthur's definition - degeneration}

The variation distribution like used in Arthur's speculation can be obtained from theoretical considerations, i.e. modelling, which by nature is only an approximation taking into account several selected aspects. The huge

\footnotetext{
${ }^{8}$ Follow (Hordijk, Altenberg 2020): "In the 1980s came the identification of "developmental constraints" as sources of information other than natural selection that shape evolution (Bonner, 1982). Subsequently, it was recognized that development also focuses phenotypic variation along certain dimensions, thus enhancing the likelihood of evolution taking these paths (Roth \& Wake, 1985). Together these have been combined into the concept of "developmental bias" (Yampolsky \& Stoltzfus, 2001)." Note, that from the beginning it competes with natural selection, however, this competition is not about source of adaptation.
} 
complexity of evolutionary conditions and the evolving objects does not give a chance to good approximation of real distributions, however, this method allows us to become aware of some of the expected properties of such a distribution.

As noted above, Arthur tacitly assumes in his model the lack of directing of changes within one feature in the distribution before selection. There is such a direction that can be expected, so when trying to indicate the simplest, as general as possible, theoretical distribution of variation before selection, it is worth considering it.

In (Gecow 2009b) I described an extremely simple model of evolution controlled only by the selection of random changes. The vector of 64 signals was subject to random changes. Each signal had s $=4$ equally probable variants and probability that it would be changed equal $1 / 4$.
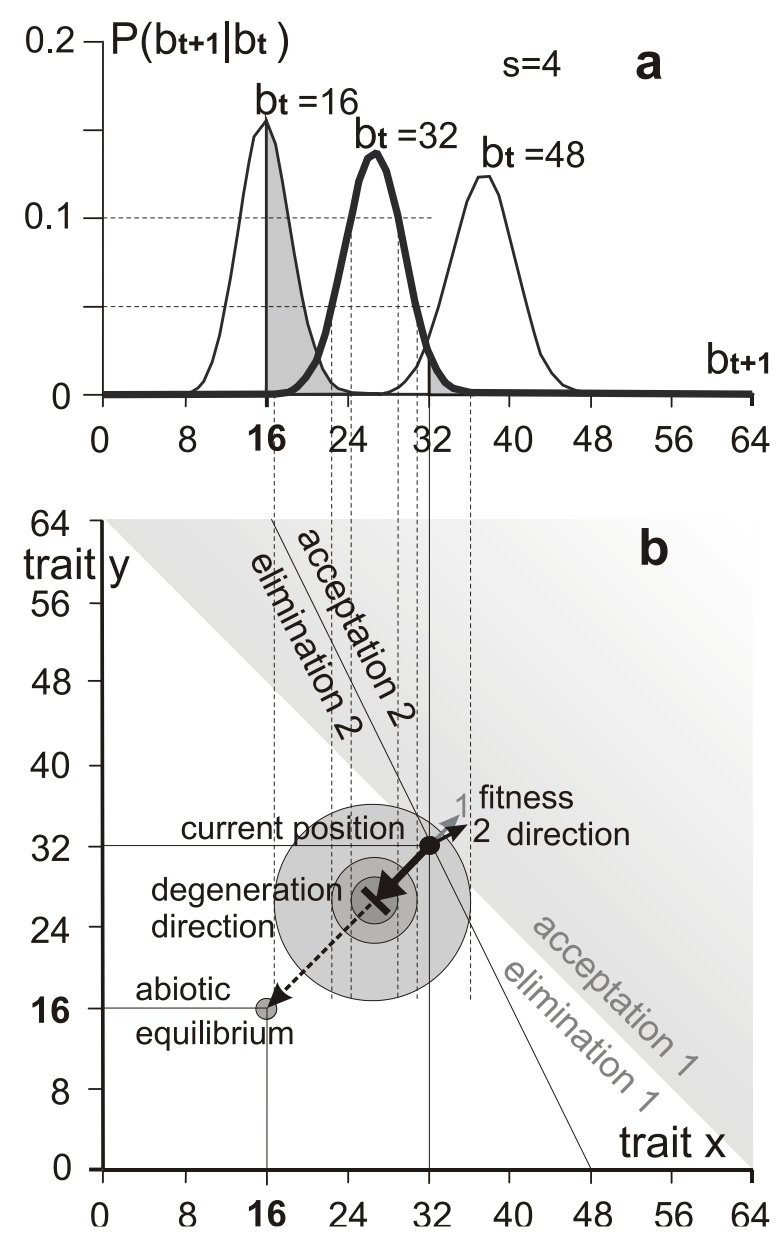

Fig. 1. Degeneration as expected bias in the simple model of adaptive evolution. The vector of 64 signals is subject to random changes. Each signal has $\mathrm{s}=4$ equally probable variants and probability that it would be changed equal $1 / 4$. Number of identical signals in the evolving vector and randomly chosen 'ideal' is called b. After each random change, the effect is compared with fixed 'ideal' vector, and if $b$ decreased, then this change not exists. Time $t$ counts only accepted changes. $\mathbf{a}$ - Three probability distributions of next $b$ after change: for $b_{t}=16,32$ and 48 . Accepted changes are shown in shaded part. Point $b_{t}=16$ can be interpreted as abiotic equilibrium - random changes without elimination do not move the object from this position. For $b_{t}=48$ the accepted part is too small to be visible. Therefore we discuss case $b_{t}=32$, there accepted part is small, but visible. Note: mean value of $b_{t+1}$ is near of top position, much lower than initial $b_{t}$, this expected move is a degeneration, it is typical entropy growth and information losing.

b - Two uncorrelated, similar to $b_{t+1}$ at stage $b_{t}=32$, traits $x$ and $y$ in form similar to (Fig. 1 in Arthur 2004). Current position ( $\mathrm{M}$ in (Fig. 1 in Arthur 2004), $b_{t}=32$ in upper figure a) cannot be in the middle. Arrow from the current position to the middle shows expected degeneration after one random change. After more of random changes (without of elimination) degeneration will move object to abiotic equilibrium. However, if requirements of environment changes, natural selection may agree for some degeneration - it is 'allowed degeneration' which take place always, when it is possible. Note, direction of further evolution depends on current requirements of environment, the may be different, than up to current time (in model they were constant). Therefore threshold of acceptance and fitness direction may also change.

Such variation distribution (just identical as curve for $b_{t}=16$ in Fig.1a) assumed in (Gecow 2009b, fig. 1.3-6) is academic and is not adequate for description of reality, however, it is the best one I have found for the theoretical investigation. Number of identical signals in the evolving vector and 'ideal' is called $b$. After each change, the 
effect was compared with fixed 'ideal' vector, and if less signals were identical (b has decreased), then this change was rejected (eliminated). Three probability distributions of $b$ after change are shown in Fig.1a: for $b_{t}(b$ at time $t)$ $=16,32$ and 48. Point $b_{t}=16$ can be interpreted as abiotic equilibrium - random changes without elimination do not move the object from this position. If selection works, then only right, shaded half is accepted. For $b_{t}=48$ this accepted part is too small to be visible. Therefore we will discuss case $b_{t}=32$, there accepted part is small, but visible.

Value $b_{t}$ can be treated as some trait measured in a unit highly correlated with its fitness value. In Fig. $1 b$ two such traits are used. We assume, that they are independent and fitness is a sum of trait's values. The peak from fig. 1a is here shown by its levels and darker shadows for higher parts. Here current position is not in the middle of the circle. Expected shift (before selection) is from current position to the middle of circle, but if evolution will not be controlled by selection, then it will lead object near to the 'abiotic equilibrium' in few steps (Gecow 2009b fig.1.3 and 1.5). This is a degeneration, it is always present when selection does not block it. It is one of the bias, which is not present in Arthur fig. 1 due to using less adequate distribution.

This extension of Arthur's view (let us call it 'degeneration model') contain important degeneration, but it is already connected to fitness direction, that Arthur in his conception had avoided. However, it is connected to fitness direction (and results of selection work) present before current distribution of changes was defined and before coming selection which will work with new fitness landscape with probably another direction. Current distribution is always an effect of past selection in past fitness landscape. Only the new direction is important for Arthur and distribution is still independent on it. Therefore degeneration is the bias. Like Arthur's bias, degeneration is a factor in the theoretical distribution of phenotypic changes before selection that affects direction of evolution.

Due to change of Wright landscape and traits correlations over time and evolution, the path to 'abiotic equilibrium' is in reality not a straight line in multidimensional morphosphere, and the current direction to it (direction of degeneration), like a current direction to fitness, often changes. In this simple model there is no collected biases which increase mean fitness of changes given into selection (we will discuss them later), which in reality strongly change distribution of phenotypic changes before selection. This lack is a reason why this figure is fare from intuitive picture of current reality. Degeneration simply explains 'domestication syndrome' discussed in (Wilkins 2020). I call such degeneration an 'allowed degeneration'. It is limited by natural selection which reduces requirements due change of circumstances. Note, however, Wilkins used distribution after selection (because only such distribution is available from experiment) to indicate bias.

\subsection{Half-chaos - a basis of the 'small change tendency'}

Added here aspect of degeneration is just one of many possible. In (Gecow 2009b) the 'small change tendency' is stressed. In above model it is present as result, but what means 'small'? It is important feature of change distribution because when limiting changes size to so indicated range, then the mean fitness will radically increase. Emerging of such limitation mechanism will create a strong bias. In (Gecow 2019b) I show simulations of model of complex network evolution where I got answer for question 'what means small change?' and 'how looks like 'full' distribution of changes before selection?'. In this distribution clear peak is visible for 'chaotic' effect of 'mutation' which is a model of death (elimination), and another peak of small changes. Between these peaks a deep gap exists that in natural way define 'small change'. In opposition to known Kauffman's hypothesis 'life on the edge of chaos (of fully random networks)' (which is used in GRN model cited in Special Issue) the results show that life evolves in 'half-chaos' of not fully random networks, which frees modelling from difficult restrictions. The half-chaos was unknown until now. By limiting of accepted changes to only small changes, the process does not leave the half-chaos allowing for a long evolution of the slowly changing system (the system retains identity, it works similarly), which creates a natural criterion for the identity of the evolving object. The acceptance of one change that gives a chaotic reaction (great change of function) leads to practically irreversible entry into normal chaos (the system works completely different, ceases to be itself). Thus, the basic Darwinian mechanism emerges - this has large interpretational consequences.

Initially, I thought that negative feedbacks and modularity are the basis of the stability of living organisms, but the simulations turned out that the short length (in time steps) of attractor plays a major role and these two factors are only the main supporting. The global attractor can even be long if it is composed of several small attractors inside non-communicating (not transmitting disturbances) modules. It is one more source of modularity, than was found in (Altenberg 2005) where role of modularity, similar to development bias, is deeply analyzed. The modularity and negative feedbacks lead to increase of expected mean fitness in the variation distribution before selection, therefore emerging of such features also creates biases.

\subsection{Deeper into theory}

To better know what it is the developmental bias we should describe it in probability language. The fitness distribution (Wright's landscape) is described by $\mathbf{P}(\mathbf{a} \mid \mathbf{X}, \mathbf{x 0})$, which is the probability of acceptance (a - life cycle 
survival) when the evolving object in state $\mathrm{x} 0$ will have certain parameters $\mathrm{x} \in \mathrm{X}$. Because the fitness distribution depends on the current state $\mathrm{x} 0$ of the object under consideration, then the dimensions of the $\mathrm{X}$ space are not so much absolute parameters, but their specific changes - a deviations from the current state $\mathrm{x} 0$ and such changes interests us more. Using the formula $\mathbf{P}(\mathbf{a} \mid \mathbf{X}, \mathbf{x} 0)$ we can always calculate the change as $\mathrm{x}-\mathrm{x} 0$, but repeating $\mathrm{x} 0$ in all formulas is inconvenient and is usually ignored, however should remain in the memory. If $\mathrm{x} 0$ is omitted, then $\mathrm{x}$ has two opposite interpretations at the same time - it is a parameter and its change. Bearing this in mind, we will simplify the notation to $\mathbf{P}(\mathbf{a} \mid \mathbf{X})$ and interpret $\mathbf{x}$ as a change of the object.

What are the dimensions of the $\mathrm{X}$ space? - anything that can change and affect the likelihood of acceptance. So, in addition to all parameters of the object itself, including morphosphere and behaviour, also parameters describing its environment should be included. However, parameters of environment and in similar way like above - their changes, as forced and independent on object and its selection, we exclude to separate set $\mathbf{V}$. Then full set of changes, that influence probability of acceptance is described as $\mathbf{Y}=\mathbf{X}+\mathbf{V}$ and simplified formula describing Wright's landscape get form $\mathbf{P}(\mathbf{a} \mid \mathbf{Y})$.

We will divide the description into stages in order to discuss more complex cases on the already known basis. In the first stage, we will only be interested in changes of the evolving object - X, and we will assume that there are no changes $\mathbf{V}$ of the en Vironment. Changes of the object appear with a certain probability $-\mathbf{P}(\mathbf{X})$.

The direction of evolution is observed as the mean $\mathbf{x}-\mathbf{E}(\mathbf{x})$ among the cases of changes that are available for observation, i.e. typically they have been accepted and in the distribution $\mathbf{P}(\mathbf{a} \wedge \mathbf{X})=\mathbf{P}(\mathbf{a} \mid \mathbf{X}) \mathbf{P}(\mathbf{X})$.

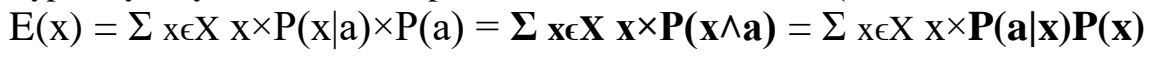

As can be seen, this direction depends on the two factors $\mathbf{P}(\mathbf{a} \mid \mathbf{X})$ and $\mathbf{P}(\mathbf{X})$ described above. The fact that the $\mathbf{P}(\mathbf{a} \mid \mathbf{X})$ - fitness distribution (Wright's landscape) influences this direction is obvious to all. Arthur noticed that $\mathbf{P}(\mathbf{X})$ also influences this direction, if it is not a flat distribution, the same for every possible $\mathrm{x} \in \mathrm{X}$, and that this influence, as a separate factor present before the work of selection $\mathrm{P}(\mathrm{a} \mid \mathrm{X})$, he called 'bias'. As an example of such a bias, Arthur gave the correlation between the dimensions of the vector x. In Fig.1a it can be seen that the probability of change for the state with increased adaptivity is much smaller for the value of $\mathrm{x}$ with greater fitness than the change $\mathrm{x}$ with lower fitness, which is also an example of heterogeneity of $\mathrm{P}(\mathrm{X})$. Here it should be remembered that this is the probability of changing the current state.

Developmental bias (hereinafter - 'devbias', see ch.3) is therefore the heterogeneity of the distribution of $\mathbf{P}(\mathrm{X})$. The presence of this factor before the selection is the main intuitive feature of the developmental bias concept. The selection occurs after the change and it takes a time, it is based on $\mathbf{P}(\mathbf{a} \mid \mathbf{X})$.

The selection period is clearly divided into stages, mainly in the Amniotes, which are the basic intuition reference for evolution. These are: development, the period until the offspring are released, and the multigenerational time to test a new feature. This will also be discussed further due to the time of observation of the effect. But the factors giving rise to $\mathbf{P}(\mathbf{a} \mid \mathbf{X})$ and its effect may already be sufficiently known, at least some, and the outcome of this selection may also be determined (at least statistically) prior to its occurrence. It is generally the theoretical predictability that our cognition seeks. Such the factors can be indicated for specific $\mathrm{x} \in \mathrm{X}$, which are also 'present' before selection and, similarly to devbias, affect the direction of evolution, although they are included in $\mathbf{P}(\mathbf{a} \mid \mathbf{X})$ but not in $\mathbf{P}(\mathbf{X})$, and are independent of changes $\mathrm{V}$ in the environment, that is, they do not consist of the effects $\mathbf{P}(\mathbf{a} \mid \mathbf{V})$. Such factors are e.g. 'structural tendencies' (Gecow 2005, 2009a) defined as general heterogeneities in $\mathbf{P}(\mathbf{a} \mid \mathbf{X})$, explaining and predicting the known classical regularities of ontogenesis evolution, such as Naef's (1917) 'terminal modification and conservation of early stages' or 'terminal additions and compression of early states of ontogeny' (Weismann 1904). From the point of view of the main intuition of the devbias concept, they are somewhat of sister phenomena to the devbias, and it may be difficult to distinguish their effects from those of devbias. To draw attention to this misleading circumstance, they will be separated in experimental approach as pseudo-devbias5.

Until now, we have mainly considered the distributions of $\mathbf{P}(\mathbf{a} \mid \mathbf{X})$ and $\mathbf{P}(\mathbf{X})$, as they have theoretical and explanatory significance, they define Wright's landscape, devbias and structural tendencies. However, the frequency of phenotypes (observed morphosphere) is the result of the observations, but not the considered distributions, which we do not know. It does not even correspond to the distribution of $\mathbf{P}(\mathbf{X} \wedge \mathbf{a})$, since $X$ are changes, not states. In order to determine changes, we must use omitted for simplicity $\mathrm{x} 0$ or have state distributions from two separate moments. Of course, this is only realistic for a few selected parameters and the entire analysis must be limited to them, assuming that the changes in the remaining parameters are irrelevant to the analysis being carried out. In such a limited scope, one can try to determine unknown elements, including the very interesting $\mathbf{P}(\mathbf{a})$ and the $\mathbf{P}(\mathbf{X})$ defining devbias. Note that after the change takes place, all the elements of this puzzle may change, because the initial state $\mathrm{x} 0$ of the object changes, so $\mathrm{Pt}(\mathrm{a})$ can also be changed to other $\mathrm{Pt}+1(\mathrm{a})$, and this is a much-discussed effect, especially when $\mathrm{P}$ (a) grows significantly (in the discussed range of parameters). The sets $\mathrm{X}_{\mathrm{t}}$ also change to $\mathrm{X}_{t+1}$, which makes the analysis difficult, but they can be summed as $\mathrm{X}=\mathrm{X}_{\mathrm{t}} \vee \mathrm{X}_{t+1}$ as they are usually very similar, and small differences are expressed in $\mathrm{Pt}(\mathrm{a} \mid \mathrm{X})$ and $\mathrm{Pt}+1(\mathrm{a} \mid \mathrm{X}), \mathrm{Pt}(\mathrm{X})$ and $\mathrm{Pt}+1(\mathrm{X})$. The last 
forms devbias in themselves. $\mathrm{X}$ is the morphosphere available from objectt and from object $t+1$ through one change, if it is not available it has probability $=0$.

Determining $\mathrm{P}(\mathrm{X})$ is also not exactly what we are looking for. Before the change is examined, i.e. in the $\mathrm{Pt}(\mathrm{X})$ with very high probability, in principle with certainty, there are already some heterogeneities (i.e. devbiases) of various origins. By focusing on a specific change $\mathrm{z} \in \mathrm{X}$ of the object, we can see this change and the resulting difference: $\Delta \mathrm{P}(\mathrm{X})=\mathrm{Pt}+1(\mathrm{X})-\mathrm{Pt}(\mathrm{X})$ occurring on some subset $\mathrm{C} \subset \mathrm{X}$, probably $\mathrm{C}$ contains more elements than only $\mathrm{z}$ (e.g. due to correlations). This difference is devbias3. It prefers something (some changes $\mathrm{x}$ ) and avoids something, and this is a concrete new devbias, the mechanism of which we can analyse. Note, that in such

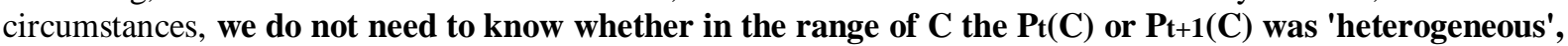
and we are not interested in whole two $P(X)$ distributions, but we are only interested in the existence of $\Delta \mathbf{P}(\mathbf{C})=\Delta \mathbf{P}(\mathbf{X})$ in a small range of $X$, which affects the direction of evolution and may affect $\mathrm{P}(\mathrm{a})$. However, we cannot estimate $\mathrm{P}(\mathrm{a})$ over the whole range of $\mathrm{X}$, maybe it will be possible on $\mathrm{C}$, but the difference $\Delta \mathrm{P}(\mathrm{a} \mid \mathrm{X})=\mathrm{Pt}+1(\mathrm{a} \mid \mathrm{X})-\mathrm{Pt}(\mathrm{a} \mid \mathrm{X})$ can appear on a subset $\mathrm{C}^{\prime}$ of $\mathrm{X}$ other than $\mathrm{C}$, though they may have a large common part. Because $X-\left(C \vee C^{\prime}\right)$ is not interesting, then from this point of article we will understand $X$ as only $\mathbf{X}=\mathbf{C} \vee \mathbf{C}^{\prime}$.

In the second stage of our analysis, we will consider changes $\mathbf{V}$ in the environment. They not only have the probability of the occurrence of $\mathrm{P}(\mathrm{V})$ and influence the survival of the object $\mathrm{P}(\mathrm{a} \mid \mathrm{V})$, but also modify the earlier $\mathrm{P}(\mathrm{a} \mid \mathrm{X})$ to the new form of $\mathrm{P}(\mathrm{a} \mid \mathrm{X}, \mathrm{V})=\mathrm{Pv}(\mathrm{a} \mid \mathrm{X})$ because $\mathrm{x}$ are assessed in another circumstances. They can also modify $\mathbf{P}(X)$ to $\mathbf{P}(X \mid V)=\mathbf{P v}(X)$ because $v$ can modify object before selection and this is a very important and discussed here plasticity which changes development without change of gene. As can be seen, this new situation is much more complex. We are still interested in change of $\mathrm{Ev}(\mathrm{x})$ in the range of $\mathrm{x} \in \mathrm{X}$ i.e. mean object change, but now it also depends on environmental changes:

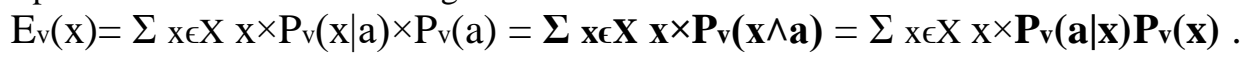

We can still mainly observe the object state distributions after and before a specific change or subsequent steps of variation, and to find devbias, we must estimate $\Delta \mathrm{Pv}(\mathrm{X})$. The awareness of what the considered effects are in a description similar to the above very simplified and provisional one, will radically facilitate and authenticate such an analysis. This will allow to notice and indicate many important assumptions usually omitted in the present descriptions of similar phenomena.

\section{Experimental approach, aspects of 'developmental bias'}

Clearly theoretical approach of Arthur, in the works following him changes to a descriptive and experimental approach attempting to put Arthur's concept into practice.

Promoted in Special Issue the term 'developmental bias' is openly and provisionally based on the meaning formulated in (Uller et al. 2018). This meaning is similar to Arthur's definition, but less exact, however, Special Issue was open for other, new suggestions. This led to the loss of the original definition and uncontrolled blurring of the meaning of the promoted term. The search for bias in real distributions, typically $\operatorname{Pv}(\mathbf{a} \wedge \mathbf{X})$ after selection, resulting from the transition from theory to practice is the first significant deviation. 'Typically' here means, that exception exist. It is a case, when phenotypic change is observed just in the time it occurs in development. It is easy to expect that such moment exist for plasticity, but also mutations exhibit their effects not on the very beginning of the development. In such the time point a selection has not yet had time to react, and measurements taken at this stage of development can be considered 'before selection', but this is a rather rare case in practice.

Direction to higher fitness is especially discussed ${ }^{9}$ and treated as typically observed bias. This is another deviation and another bias which needs another definition and should be distinguished from Arthur's concept. These are not the only deviations.

Maybe this brainstorming method is useful, but without the theoretical ordering of the collected material, it will destroy Arthur's sensible concept. E.g. as result, in Wikipedia there is unclear description ${ }^{10}$, that has nothing to do with definition. This lack of clarity of the term 'developmental bias' has forced the search for the meaning also directly from the meaning of both words used at that term, whose meaning seems understandable. This was the reason for the further uncontrolled increase in the scope of the concept and the change of emphasis on 'illegally' introduced meanings. In order to reduce the basis of this path and force the definition to be used, in the new situation resulting from the presented analysis, I propose to replace the lengthy two-word term with a new, shorter

\footnotetext{
9 "As a form of adaptive plasticity that allows organisms to shift their phenotype toward the optimum, learning is inherently a source of developmental bias.” (Laland et al. 2020) .

10"In evolutionary biology, developmental bias refers to the production against or towards certain ontogenetic trajectories which ultimately influence the direction and outcome of evolutionary change by affecting the rates, magnitudes, directions and limits of trait evolution." Wikipedia [March 30, 2020]
} 
term 'devbias'. It will stabilize the position of the term in the Special Issue intention, which I do not specifically support, but I accept, provided that the definition is used.

So first of all we should analyse the suggestions contained in (Uller et al. 2018).

\subsection{Theory versus experiment, before or after selection}

Getting to apply or test the theory in practice, we must realize that theoretical entities, necessary for a simple understanding of the relationships under consideration, may be difficult or even impossible to observe directly in practice. In the Arthur's definition it is important, that distribution (of object variations, $\mathrm{P}(\mathrm{X})$ ) concerns variation before selection - it allows to call defined phenomenon a 'bias' as some feature, that influence later emerging direction of evolution.

However, such distribution before selection is hard to measure - except the time point of occurrence of a change in development there is no changed phenotypes that are before selection. We can define some points in the development, that are naturally indicated. For vertebrata 'later' than fish, it may be first not larval stage in amphibians or moment of hatching from the egg or birth in mammals. From the time of change appearing (e.g. mutation) to this time-point embryo develops and of cause - selection works. Let us call it 'small-selection'. The second such point may be the time of reproduction. Let us call it 'middle-selection'. But we observe evolution in time measured in lot of generations, in fossil data or even in laboratory experiments. Then selection in such long period should be called 'great-selection'. All these four type of selections give other distributions and different biases which should be distinguished.

The division of currently discussed set of 'devbiases' into separate boxes suggested in the Introduction must be based on some aspects that significantly affect the properties of these 'devbiases'. Above, we found two such aspects, these are the way to indicate a given bias which can be theoretical (' $\mathbf{t}$ ') or experimental ('e') (including observation), and the time relative to the selection to which the distribution of variation in which this bias is indicated relates. I suggest, that taking this into consideration, we should add to given devbias a letter indicating such circumstances. For example in the second aspect: b-before selection, at least a-after selection or better instead ' $a$ ' (if it is known and important) more exact: s-small; m-middle or g-great selection.

Before considering the use of such notation in examples, we will look for further relevant aspects and variants that they adopt.

\subsection{Five problems}

The term 'developmental bias' is constructed for problem (let us call it 'problem1 A')-influence on direction of evolution (in fitness space, based on natural selection) of correlations of phenotype change parameters (as factor independent on current fitness landscape). For this problem Arthur (2004) defined 'developmental bias' (devbias1A) as extension of concept of 'developmental constraint' (against a certain ontogenetic trajectory) by adding 'developmental drive' (towards a certain ontogenetic trajectory). They are treated as two types of 'developmental bias', but I am going to split currently used such the term in another aspects into set of types.

I have extend this theoretical problem removing from its limitation to correlations, then it covers now also directions in phenotype change parameters also as factor independent on current fitness landscape. Such extended problem, still looking for influence on evolution direction, should be called 'problem1' and found factors generally - devbias1. Degeneration (ch.2.2) belongs to such category. Both - devbias1A and degeneration (devbias1D) are shown using theoretical speculations (denoted by ' $t$ ') and clearly before selection (denoted by 'b'). However, Wilkins' (2020) 'domestication syndrome' is an 'experimental' observation ('e') which is after selection ('a'), then it is devbias1Dae and it differs to devbias1Dbt described above.

Arthur's formulation of the promising theoretical term 'developmental bias' creates a natural need to use and verify it in practice basing on the given theoretical definition. This is a problem2. However, it meets two important obstacles: The first is the above indicated deep difference between the variation distribution before selection $(\mathrm{P}(\mathrm{X}))$ used in the definition, and the distribution after selection $(\mathrm{P}(\mathrm{X} \wedge \mathrm{a})$, see ch.2.4), typically available for experimental measurement. The second is the simplicity of the model available for modelling and theory (to obtain $\mathrm{P}(\mathrm{X})$ ) but unavailable in observing real objects that are extremely complex. First of all, we expect a huge accumulation of various biases in real living objects, and separation of the correlation that creates the studied bias requires comparison of similar distributions rather than analysis of one experimental distribution. Of course, such an analysis of a specific bias must concern at most a few selected change parameters, but limiting ourselves to a sufficient set of changes that affect the considered effect is difficult. Such a comparison of two real distributions (typically after selection $\Delta \operatorname{Pv}(\mathrm{a} \wedge \mathrm{X})=\mathrm{Pvt}+1(\mathrm{a} \wedge \mathrm{X})-\mathrm{Pvt}(\mathrm{a} \wedge \mathrm{X})$ ), may be the basis for indicating a hypothetical bias in the distribution before selection $(\Delta \mathrm{Pv}(\mathrm{X})=\mathrm{Pvt}+1(\mathrm{X})-\mathrm{Pvt}(\mathrm{X}))$, but this is a completely different problem3.

In natural way it is noted, that some such hypothetical biases direct evolution to higher fitness and new 'problem4' emerges - are resulted adaptive evolutionary changes an effect of Darwinian natural selection or such devbias4 is an additional, independent source of adaptations? Bias connected to this problem should 
be called devbias4, but it is also 'devbias3+'. Such symbol ('+') means, that distribution with such devbias3+ has higher mean fitness (in the range of considered parameters) than distribution without it. Answer in short: Emergences of this devbias, as any other feature changes, are created as blind ${ }^{11}$ on needs. On this stage it is not yet assessed by selection, it is under ('@’) selection. Let us denote it by sign '@, which give devbias3@. But Darwinian natural selection cut out 'not useful' cases. Useful ${ }^{12}$ cases remain (as devbiases3+ equal to devbiases4 selected from devbiases3@) and then in current evolution (considered period of evolution) help to propose changes of higher probability to be adaptive. However, natural selection of devbiases3+ (devbiases4) from devbiases3@ is the source of such adaptability. Devbias4 is a go-between only, not an independent factor creating adaptation. To state this openly is the main aim of this article. As especially important the problem 4 with devbias 4 are separated from the problem 3 as distinct. Evolution collects devbiases 4 then experimental distributions are full of devbiases4 (as was noted while discussion of degeneration) and this disturbs investigations in problem2. To complete the set of devbias 3 in aspect of mean fitness of new distribution we should add case, where mean fitness decreased - obviously it should be denoted by '-'.

Problem 2 was discussed and it seems to be hanged due to troubles that should be explained. In next chapter I pick up this discussion. However, it probably will disappear (in the maim part) and the main problem taking under consideration experimental data will remain problem3. It contains two recognized sub-problem 3 which should be separated, one (problem4) because it importance and one (problem5) because it gives pseudo-devbiases.

Both problem 1 and problem4 are different, but it is easy to mix them. Problem 1 leads to statement, that "variation is not random" (in the sense - they are anisotropic, $\mathrm{P}(\mathrm{X})$ is not flat), but the same wording in the problem4 has another default meaning (in the Darwinian sense - they are not blind on needs). To discuss problem 4 a clear distinguishing of both problems is necessary. However, distinguishing of problems is not easy in practice, and they may overlap in part. The 'domestication syndrome' at first stage after observation may be treated as devbias3ae with controversial, not known assessment of fitness change direction. It is mainly due necessity of comparison of two distribution for observation. I classify it rather as devbias1ae (devbias1ge) which is connected to devbias1Dbt. Typically found devbias3ae gives only hypothetical devbias3bt which is interesting, searched devbias. Theoretical explanation should be a next and the last step but it may differ not much to devbias1bt. Devbias3b+ makes higher mean fitness of current distribution before selection (in the range of considered parameters), but it is higher in relation to earlier distribution which does not contain this particular devbias $3 b+$. The current distribution $\left(\mathrm{Pt}_{t}(\mathrm{X})\right)$ is still random and blind on needs, it only looks as biased from previous point of view (at $\mathrm{t}$ ) which differ to current one.

I have found one problem more for this collection - 'problem5' is when experimental data investigated in similar way to problem3ae give strong regularities $\Delta \operatorname{Pv}(a \wedge X)$, but they are not a result of some hypothetical devbias3be (as $\Delta \operatorname{Pv}(\mathrm{X})$ ). Such tendency may be found and explained also theoretically, but they exist only after selection. Kavanagh (2020) had noted: "When condensations are highly integrated and dependent on each other during early organogenesis, both plasticity and evolution are restricted, ... When non-similar structures form separately and then integrate while still undergoing patterning, high levels of plasticity or new dimensions of ecologically-significant variation are seen.". Such observations are just Naef's (1917) regularity of "terminal modification and conservation of early stages' in (too) simply way explained by de Beer (1940), and now, using simulation of complex networks, in (Gecow 2005, 2009a). In my simulations I have observed more such classic regularities of ontogeny evolution as simple result of selection and complexity. "Without the inheritance of acquired characters, Weismann's proposition of "shunting back theory" ("pressing back theory"; later version of Haeckel's conception) - terminal additions and compression of early states of ontogeny (Weismann 1904) lost (Holmes 1944) one of its pillars. Weismann's concept was transformed by Naef (1917) to that of terminal modifications and conservations of early stages." (Gecow 2005). In these simulation the inheritance of acquired characters was not used and changes are drawn randomly $\left(\mathrm{Pt}_{t+1}(\mathrm{X})=\mathrm{Pt}(\mathrm{X})\right)$. These regularities are an effect of selection (they are ready in $\operatorname{Pt}(\mathrm{a} \mid \mathrm{X})$ ) and while observation they look like devbias3ea or more exactly - like devbias3eg (in the case of observations of $\Delta \mathrm{Pv}(\mathrm{a} \wedge \mathrm{X})$ made by e.g. Haeckel). Because their distribution of change before selection does not change they are not devbiases. In the case of my theoretical and simulation investigation I have study $\mathrm{P}(\mathrm{a} \mid \mathrm{X})$ that become not flat and I call them 'structural tendencies'. When they are observed in reality, they should be taken provisionally as devbias3ae, however, because there is no devbias $3 \mathrm{~b}$ they are not normal devbiases, they may be treated as pseudo-devbiases and should be distinguished from devbias3. For them it remain name pseudo-devbias5.

\footnotetext{
11 The term 'blind on needs' is not so obvious as can be expected. It is discussed in (Pocheville, Danchin 2016) but I have another view which I discuss in ch.6.3.

12 Neutral cases also remain, but we ignore them here.
} 


\section{Comparison of theory and observation - problem2}

Arthur made theory, which indicates the promising term 'developmental bias'. This provoke to check how works such theory on real data and how looks like real devbiases? This is the next problem after theory formulation, we have called it problem2, then biases found in reality, identified using Arthur's definition should be devbiases2. In (Uller et al. 2018) the first period of investigation is summarised. Theoretically predicted 'possible phenotype space' plays an important role in the definition. Let us at the beginning improve our intuition of meaning of this term.

\subsection{Intuitive abstract examples}

Let us now consider meaning of term 'developmental bias' and 'possible phenotype space' basing on Arthur's definitions and using a simple theoretical example.

Suppose we have 3 features $x, y, z$ describing the phenotype. Currently, each of them has a value of 1 . Mutations can increase each of them up to 8 times and we believe that such mutation are equally probable. So we have a space of possible (in the sense-1) phenotypes with $n=512$. When each of them has the same probability, there is no bias. So: Until we do not know the connections between the measured features, we expect an even (isotropic) distribution which means that there are no such connections (correlations). If it turned out to be like that, there is no bias.

If $x$ is a length, $y$-muscle force, $z$ - mass, then it is known that when the growth is proportional and the length $x$ increases to $x=2$, then the muscle force $y=4$ and mass $z=8$. But if $x=4$ then $y=16$ and $z=64$, which does not meet the above assumption about the range. We can assume that even $x$ may increase to 8 as a result of mutation, but with a weight of $z>8$ and these proportions, the animal is too heavy and cannot survive. The range of possible directions of changes is therefore also determined by natural selection. This cuts the ranges of available phenotype space. Now (if our measuring accuracy is limited to whole numbers) $n=2 * 4 * 8=64$ (in the sense1). Is this cut already the real, objective bias? - Probably - no, it results from the adopted units and ranges at previous step.

If $x$ can take any (measurable with higher than previously resolution) value in the range $0.5 \leq x \leq 2$ with equal probability, it will be a curve almost continuous in three dimensions of space: $(x, y, z)=\left(x, x^{2}, x^{3}\right)$. It gives very strong covariations and in intention of Arthur it is certainly a bias visible in more exactly described space (large $n$ but still in the sense-1). It is devbias1. Was it allowed to take such strongly related features? - When entering the study, we usually do not know if and how the indicated features are related. Here, I intentionally used related features in a known and obvious way. And if it was not allowed, what connections are inadmissible and how are they different from those used here?

There will be some blur in real measurements, but this result will not take up all the available, already cut space, the probability distribution in it will not be even. This is also devbiasl. However, we know why remaining part of phenotype space is empty and problem emerges - have we correctly indicated available phenotype space? Maybe, we should subtract those phenotypes in which there is no curve $\left(x, x^{2}, x^{3}\right)$, or blur gives probability near 0 (here arises problem where probability is 0 exactly). Then we will limit ourselves to really possible phenotypes (in the sense-2). In such the case questions emerges: What do we compare with to say that devbias1 exists? If they are to be determined by the probability difference in the range of probability $>0$, then what would the lack of bias look like, especially for cases with probability almost equal 0 ? However, such questions are wrongly formulated - for devbias1 available phenotype space should be only in the sense-1, it is not 'real' distribution.

Devbias1A is a real feature of probability distribution of changes from current phenotype to available phenotypes (in other words: of possible directions of changes). It really influences the direction and outcome of evolutionary change. It works in the range of problem1 defined above. Understanding it as effect of correlations of changing features is correct and useful.

However, word "bias" and statement "variation is not random" suggest something more. For example, as a result of the regulations, the force $y$ must rather increase faster than $x^{2}$ to handle mass $z$ growing as $x^{3}$, and it does it which further increases muscle mass and in addition $z$ as a result. Only such deviations of the mean value from the curve $\left(x, x^{2}, x^{3}\right)$ are the suggested real devbias4, but it concern problem4. When in the range of problem1 "variation is not random" means, that distribution of available changes is not flat (anisotropic), then in the range of problem4 it means, that variation prefers higher fitness than in the case without devbias4.

\subsection{Devbias2 as a subjective phenomenon}

In (Uller et al. 2018) the definition of 'developmental bias' is more adapted to the task of comparing with observation, however, it is based on definition given by Arthur (2004). Instead of monitoring of direction deviation in the fitness space as the effect of factor (e.g. correlation) before selection, like in Arthur theory, more attention is put to monitoring of changes in phenotype space possible to detect typically when already selection is done. At the very beginning of the abstract we read: "Phenotypic variation is generated by the processes of development, 
with some variants arising more readily than others - a phenomenon known as "developmental bias." "which is used as definition also in Special Issue. This is comparison of two distributions - theoretical ( $\mathrm{P}(\mathrm{X})$, "possible phenotype space', which should be evenly filled for case without bias) and measured (typically after selection $\mathrm{P}(\mathrm{X} \mid \mathrm{a})$ or $\mathrm{P}(\mathrm{a} \wedge \mathrm{X}))$. However, in this definition aspect of independency on selection is lost - it not indicates when 'variants arise more readily'. Presence of the factor creating bias before selection and its independency on selection was the main characteristic of the bias, but observation before selection of real distribution of phenotypic variation is exceptionally possible.

Such change of definition includes new suggestions and leads to new interpretation. Moving source of variation from genes to developmental process is the first suggestion, that connects such view to 'development-first' approach of West-Eberhard (2003) and Lamarck's acquired characters. It is nothing wrong, although a problem of inheritance arises, but it should be noticed.

This is a careful wording dedicated to the description of the observation. However, it bases the phenomenon 'developmental bias' presence on the difference of the observed distribution and the expected one which is flat. Moving from observation to its interpretation and explanation, one must first determine the objectivity of the phenomenon, and this depends here on the objectivity of the expected distribution. However, there is a serious problem with this.

The expected distribution is described by the phenotype space ${ }^{13}$ and it is noticed that this space in experiment is not evenly filled. As an example of such unevenness (bias) the similarity of the left and right limbs is indicated ${ }^{14}$. This is a good example of devbias2s because it indicates the relationship between the selection of the expected distribution and the researcher's intuition. The intuition, however, depends on experience - a good programmer would be surprised if the observed distribution indicated that the high similarity of the left and right limbs is an exception. He would expect that there is one limb construction procedure slightly modified by the left/right parameter ${ }^{15}$. This example is simple and clearly suggests that the expected distribution is strongly dependent on the pending, i.e. is subjective. This does not mean that the phenomenon of devbias 2 is only subjective and does not objectively exist, but (when defined as above) that it is an indication of the need to correct our intuition, i.e. understanding these processes ${ }^{16}$. Such understanding was given already by models that predict well, e.g. the shape of teeth in mammals ${ }^{17}$.

Interpretation of the probability has long had two variants: frequentist probability and subjectivist Bayesian probability. When we want to estimate the probability distribution, and we do not see differences between the elements of the set of elementary events that could affect the probability of their occurrence, we assume that they have equal probabilities. However, if in the experiment we find a deviation of the

\footnotetext{
${ }^{13}$ As one of many examples, in: 'Table 1 Evolutionary questions that the study of developmental bias helps to answer' there is: "Why do phenotypes occupy only a small region of possible phenotype space? Chance and the adaptive demands of natural selection combine with regulatory epistasis in evolving networks to leave only a fraction of possible phenotypes reachable (Wagner 2011)." (Uller et al. 2018).

Jablonski (2020) considers various methods of determining the morphosphere, but even theoretical ones do not assume isotropy - these are real distributions.

14 "the regulation of the tetrapod limb creates developmental bias in the number and distribution of digits, limbs, and segments (Alberch and Gale 1985; Wake 1991), and in the proportion of skeletal parts (Sanger et al. 2011; Kavanagh et al. 2013)." (Uller et al. 2018).

15 “... integration of vertebrate limbs (Hall 2015). Left and right hind limbs share developmental pathways, and mutations in a gene regulating bone growth will therefore usually affect both limbs, making them grow equally. By preventing the expression of variants with longer limbs on one side of the body,... regulation of limb development promotes variants in directions likely to be functional, even under evolutionarily novel conditions (e.g., Standen et al. 2014)." It is similar to: "Mathematical analyses of the morphospace of bird bills and experimental manipulation of bill growth indeed demonstrate that much of the observed diversity in shape can be explained by changes in only a few parameters that describe regulatory interactions among key genes (Campas et al. 2010; Mallarino et al. 2011; Fritz et al. 2014), suggesting that much of the remaining parts of morphospace is empty as a result of how bill development is regulated." (Uller et al. 2018). Such results show, that 'developmental bias' theme leads to explanation, despite phenomenon may be subjective, however, this example will occurs objective in part.

16 "Natural selection cannot work with imaginary phenotypes, only those realized by developmental systems." (Uller et al. 2018).

17 "Tooth morphology in mammals provides a particularly compelling example of how developmental studies can be combined with computational analyses to demonstrate bias. ... models were able to reproduce accurately variation in teeth morphology observed within species (Salazar-Ciudad and Jernvall 2010), predict morphological patterns both across species and in teeth cultivated in vitro (Kavanagh et al. 2007; Harjunmaa et al. 2014), and even retrieve ancestral character states (Harjunmaa et al. 2012). ” (Uller et al. 2018).
} 
measured frequencies from the expected ones based on the distribution thus estimated, we conclude that there are some differences, but we did not see them and should be sought. These differences relate to the draw procedure.

While in the case of finite sets of elementary events, estimating the distribution, with sufficient knowledge of the procedure and characteristics of the elements gives good results (e.g. thermodynamics of gas or gambler games), in the case of continuous sets there are fundamental problems known ${ }^{18}$ for over a century. So the expected distribution of phenotypes, necessary to designate devbias2, as estimated on the basis of scanty knowledge of the drawing procedure, is burdened with a significant error, what we know. Arthur, as the author of the devbias1 definition, openly admits that isotropic distribution is not an expected ${ }^{19}$, real distribution (for real objects). Others also agree ${ }^{20}$ with this, although they understand bias more broadly. The devbias 2 phenomenon defined in this way is therefore subjective and has value as an indication that we do not know all about the procedure of the drawing and the properties of drawn phenotypes and we should look for what. It is not an indication that the draw is 'fake' and has some imposed directions, e.g. for the preference of adaptation.

Let us consider an extremely simple example to address the problem of the reality of the devbias 2 phenomenon. Is the phenomenon: 'in a roll of the dice never been observed 8' subjective or objective? This is an objective information, but it does not say whether the draw is biased. It changes our knowledge about the properties of drawing a dice (if we do not have it), it has only subjective value. Objectively no bias was observed here. You can note, that 8 is not 'available' what definition requires. - Right, you may complicate the example and use available, but low probable state ${ }^{21}$ and you will get the same conclusion: experiment shows real distribution. In this sense, developmental bias 2 defined in the above way is subjective. It is not a bias which makes effect another than random. It doesn't even have anything to do with correlation.

Summarizing of given definitions and the authors understanding of the term 'developmental bias', it can be stated that this term covers a very wide range of phenomena noticed from the point of view of the observerresearcher and combines this collection into a non-coherent whole. The definition is based on discrepancy between observations and expectations resulting from the current, very simple vision of how phenotypic changes are drawn, the vision basically only intuitive. Therefore part of defined phenomena is subjective, however useful. Another part may have an objective character, similar to general intuition. To try to sort it out, we must first separate the objective part, then divide it into emerging mechanisms and describe each one separately.

\subsection{Misunderstandings resulted from problem2}

In problem2 to identify devbias2 a real (experimental) distribution of phenotype after change should be compared to theoretical distribution. This makes a question: is such devbias 2 ' $t$ ' or ' $e$ '? There is no good answer and this attribute cannot be used for devbias2. For all other devbiases attribute t/e concerns simultaneously both compared distributions.

Devbias2 usually has two disadvantages: The first - Other distributions are compared - theoretical before selection and measured after selection, which may have already modified it. This disadvantage can be overcome when the measured distribution relates to the moment when changes occur in development and therefore it can be considered before selection. Attribute b/a indicate here only measured distribution, however correct case devbias $2 b$ is probably especially rare, then typical devbias 2 is devbias $2 a$, which is incorrect.

The second - In the basic version of devbias2, the theoretical distribution is subjective assuming the distribution is flat. More generally - because it is inadequately simple, including not taking into account the

18 "The Bertrand paradox is a problem within the classical interpretation of probability theory. Joseph Bertrand introduced it in his work Calcul des probabilités (1889) as an example to show that probabilities may not be well defined if the mechanism or method that produces the random variable is not clearly defined." https://en.wikipedia.org/wiki/Bertrand_paradox_(probability) [Nov 28, 2019]

19 "It is immediately apparent from the almost infinite number of variable-probability patterns, contrasted with the single equiprobable pattern, that developmental bias is likely to be the rule rather than the exception in all species of multicellular organisms. However, ubiquity of occurrence does not ensure a directional evolutionary effect." (Arthur 2004).

20 "When viewed from the vantage point of developmental mechanisms, it is clear that the processes that produce the physical structures of organisms will also structure the phenotypic variation that results from genetic and environmental variation. The idea of "unbiased" phenotypic variation is, from this vantage point, as sensible as the idea of "unstructured development" - which of course, makes no sense at all." (Hordijk, Altenberg 2020).

${ }^{21}$ Let's rub flat opposite vertices of the cube so that placing on such vertex the cube on the table is really possible. Let's call one of them 7 and the other 8. Let now after throwing the cube the probability of staying on one of these vertices is 0.0001 , it is much more than staying on the vertex in an unstable equilibrium when the vertex is sharp and rounded. Should such states be considered 'available'? No one will consider them equally likely with the main six. 
expectations of many accumulated devbiases. This disadvantage can be overcome when there are only few accumulated devbias in the parameters under consideration, and the theoretical model predicts the measured results well enough.

It may happen, that there is no bias. Searching for such unbiased distribution the first coming idea is, that it is distribution of changes blind on needs. It is typically understood that blind on needs means 'random'. It is not the same, which leads to misunderstanding, see ch.6.3. This is a reason, why attempting to realise problem2 leads to such conclusion: "That phenotypic variation is unbiased has ... probably been the default assumption in evolutionary theory. ... this assumption is likely to be unfounded." (Uller et al. 2018). This is a basic suggestion expressed in the abstract. It can be read as undermining the basic explanation given by Darwin of the origin of purposefulness (adaptation) in the construction and operation of living entities, which raises understandable resistance.

It is really false, 'that phenotypic variation is unbiased', but it is false too, that it was 'default assumption in evolutionary theory', because it means, that such assumption is necessary and have to be fulfilled. Suggested default in other words was, that variation is 'blind on needs' but it is conscious simplification of the wording. More exact - that variation can be blind on needs, but it does not have to. It means, that it can be biased, but it is not necessary to create adaptation. Assumption that variation is unbiased was often taken as working simplification for modelling, but it was obvious also for Arthur, that it is only large simplification.

The possibility of adopting such an understanding (undermining Darwinian explanation) of the message, which the authors (Uller et al. 2018) certainly do not want to promote, is not blocked by the phrase: "Developmental bias and natural selection have often been portrayed as alternative explanations, but this is a false dichotomy: developmental bias can evolve through natural selection, and bias and selection jointly influence phenotypic evolution." This is a very correct and cautious opinion of a researcher - a phenomenologist who makes sure not to say too much, i.e. something that he cannot prove, although he believes it. Although this is one of the most clear declaration of the lack of necessity for participation of supernatural forces, which I have found in this field in literature, the enthusiasts of such the option are left with a not completely closed gate. They can understand this sentence as follows: The mechanism (devbias4) that creates evolutionary adaptive changes can be constructed through natural selection, but it does not have to, and the natural selection only allows to remain for the result of this mechanism. So adaptive evolutionary change can be constructed without natural selection (see (Laland et al. 2020) wider discussed in ch.6.1). Such an option, in the face of the possibility of explaining its origin through natural selection, is cut out by the Ockham's razor and this does not follow from observations, so the phenomenologist has not to state this, but the theoretician should add that there is no such need, and this is not the case here which gives followers of Intelligent Design a strong tool to hand.

So how should we understand these quotes? - They refer to the current, advanced evolution taking place. This evolution has earlier accumulated many devbiases4 (as currently working mechanisms) that are now really directing evolution together with natural selection of variability blind to needs. This has not been seen before. But these accumulated devbiases 4 are the result of previous evolution, when such mechanisms have not yet arisen and only the blind variability 'assumed' in the Darwinian mechanism has created them. This is exactly the same step 1 (creation of plasticity before they work) that was discussed in (Gecow 2019a) and is not sufficiently stressed in debate on 'inheritance of acquired characters'. In (Uller et al. 2018) this stage is also not sufficiently strongly emphasized, which generally results from the biologists' habits to limit themselves to describing currently observed phenomena. However, this suggests incorrect picture and leads to unnecessary misunderstandings. Lack of such stress is visible 'at first look' in (Uller et al. 2018) and in Special Issue. Reading them more detailed all is correct, e.g. using simulation Draghi (2020) studies how the complex selection pressures across a heterogeneous environment creates plasticity which is one solution to environmental heterogeneity and the main example of the devbias.

Searching for experimental devbias using method implying by problem 2 turns to be not the best solution. We already know, that formula found in problem 3 is better. Defeat of problem 2 may be treated as defeat of theory in biology, but it is wrong conclusion. Without Arthur's theory we will not search for a devbias at all. I prefer more theoretical path through looking for mechanisms of particular phenomenon, but I agree, that before it we must agree, that such phenomenon exists.

'Domestication syndrome' discussed in (Wilkins 2020) I suggest to be devbias1Dge. For his impact to developmental bias Wilkins has problem with definition - he begin his 'Introduction' by statement: "The term 'developmental bias' lacks a standard textbook definition ..." and does not cite Arthur. However he knows, that 'domestication syndrome' is not an effect of selective pressures ${ }^{22}$. Probably now he can agree to use the 'allowed degeneration' bias from devbias1Dtb for explanation. However, questions arise - why it should not be

\footnotetext{
22 "outcomes, however, can always be argued to be the product of similar selective pressures. To make a strong argument for the role of developmental bias, therefore, it would help to have examples where repeated similar changes involving traits of little or no selective value were produced in different lineages." (Wilkins 2020)
} 
devbias2Dge? or: why each devbias2 using definition of devbias1 is not simply devbias1ae. I separated problem2 as a problematic method, where theoretical distribution before selection $\mathrm{P}(\mathrm{X})$ is immediately compared to experimental distribution after selection $\mathrm{Pv}(\mathrm{X} \wedge \mathrm{a})$ for indicating devbias2. For 'domestication syndrome' indication the comparison of two real distributions as in problem 3 was used, then it is base to include it to devbias3ge, but theoretical explanation is simply found using problem1, and mechanism has no attribute one of $\{+,-$, , $\}$ which, I think, is adequate for remain devbias3. This case is relatively simple, it is not disturbed by the complexity of accumulated devbiases4, which makes it easier to classify it as devbias1D. The theoretical explanation in devbias 3 cases, although it must have a similar structure, must be able to become independent of the complexity of the accumulated devbias4, and this makes a fundamental difference.

\section{Comparison of two real distributions - problem 3}

\subsection{Devbias3 as an objective phenomenon}

Knowing the typical cube (its required distribution), when we state after many throws that "never been observed 2 ", we can say that this is an objective deviation from the normal state, i.e. the draw is objectively biased. We guess that on the wall where there should be 2 is something else. Examining obtained results, we find, for example, that 6 has a frequency of $1 / 3$, so we expect that instead of 2 there is 6 . Because it is objective, we demand cancellation of the game and a commission examination of the cube.

Then, not all cases of devbias 2 are merely subjective, undoubtedly some are objective phenomena. To separate them, we must replace in the definition the 'expected distribution' by a 'real (not expected only) distribution'. It largely changes of definition which change the main element of problem2, and create new problem3. It leads to different method of indication of devbiases which cease to be devbiases 2 and become devbiases3.

Devbias is a feature of distribution of changes of development. Statement that current distribution exhibits lot of correlations and devbiases is obvious and trivial. Now we look for a new particular correlation and devbias. Changed definition describes another than devbias1bt, real devbias3ae, based on experiment and observation, however in the range of used understanding of 'development bias'. In the first stage devbias3ae is only a hypothesis that it is devbias in Arthur sense and connected to it devbias3bt exists. To prove such hypothesis in practice we can indicate the mechanism that creates devbias3ae effect, then compare the distribution without and with this mechanism. To state, that real new devbias3ae emerges, we have to compare earlier and later distribution of the same line. The obtained difference ${ }^{23}$ is indisputably objective. This difference is the normal result of evolution by natural selection.

\subsection{Mean fitness aspect}

We should remark, that we discuss change of probability distribution. Such distribution change may be connected to some 'current change' of phenotype (or more detail - of development i.e. ontogenesis), and this current change is assessed by natural selection relatively fast. Let us assume it is not eliminated. But it will give effect in future in next changes, and this additional effect (real devbias) is not assessed yet. Further assessment may be diverse. Until this test is going on, new distribution is a devbias3@, it may be better or worse in fitness sense, it is random (especially in Darwinian sense - blind on needs) as any tested new feature. After long time needed to asses new distribution and if circumstances do not change, a verdict is ready - if owner of such distribution still exists, then this devbias3@ turns to be a devbias3+ (devbias4) ${ }^{24}$. Only devbias4 can produce 'not random changes' which are more readily adaptive (than in previous, real and objective, but currently already not working distribution). In the current, working distribution they are still random and blind on needs. The devbias4 is not a new source of the adaptations, that are independent on Darwinian mechanism, it is only go-between, intermediary because Darwinian mechanism has created it before.

Most of biases found in investigation of the fossils (Jablonski 2020) are devbias4ge. However, biases coming from plasticity (limited to developmental adaptive reaction on environment changes) are probably typically devbiases4s and are typically not heritable yet, before genetic assimilation (in West-Eberhard (2003) sense, see

\footnotetext{
23 "Within lineages, the evolution of novelties, such as shells, limbs, photic organs, feathers, wing patterns, or horns, is associated with rewiring existing developmental building blocks and processes into new regulatory networks. This predicts that, once they appear, diversification of novelties should proceed rapidly at first, and slow down as their regulation becomes developmentally entrenched. Consistent with this prediction, the shape of bird bills diverged rapidly during the early radiation of modern birds, and subsequent evolution of bill shapes within major bird lineages has been filling up only limited parts of morphospace (Cooney et al. 2017). " (Uller et al. 2018).

${ }^{24}$ Neutral effect is included to devbias3+ (devbias4).
} 
(Pocheville, Danchin 2016)). Developmental plasticity (in wider meaning) is understood in $\mathbf{P v}(\mathbf{X})$ as

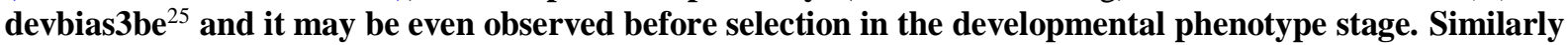
as devbias3be can be observed effect of mutation when its first occurrence is enough late in the development.

If the current change (with resulted new distribution) is coherent with (sensitive on) the regularities of the environment, then it may be a change creating in the future better proposals ${ }^{26}$ for the next changes, which will more often be adapted to the requirements of the environment. Assessing a new distribution requires more time than a single change. Suppose it turned out to be worse (devbias3-) and a rating of the current change was positive, although for this change it was necessary to give up some good property, but rarely used. This is specialization leading to 'goat's horn'. Then in long perspective real devbias3 may be a negative ${ }^{27}$ change, but the return is all the more difficult the more of subsequent changes were based on the presence of this current change. I have simulated (Gecow 2005, 2009a) this regularity by describing ontogenesis as a randomly growing network. This theme, including status of such regularities, is discussed in the range of problem5 in ch.3.2.

The mean fitness change (in the range of considered parameters) of new distribution of phenotypic changes prepared before assessment by natural selection is treated as the main parameter of the change of old to new distribution which explains why this change remain in the evolving object. I suggest, that devbias 3 should be limited to phenomena with defined such attribute (one of $\{+,-, @\}$ ). The 'pseudo-devbias5' is different than devbias 3 also due lack of this attribute. The case of devbias3ae+ is especially important and leads to wide debate of another problem4 based on this higher mean fitness, therefore it is excluded as devbias 4 .

\section{Problem4 - source of adaptation}

In the article (Gecow 2019a), I focus on the sources of adaptive changes. This theme comes back together with the phenomena admitted in the last two decades to a wider discussion and leading to the EES $^{\mathbf{2 8}}$ proposal by Jablonka and Lamb (2007, 2008). The wording "variation is not random" (Laland et al. 2014) well indicates these phenomena, however it covers all three separated meanings of randomness (see ch.6.3), typically Darwinian meaning is taken. The theme is not especially stressed, but it is crucial and needs clarify. For this the methodology should be corrected - put more attention for definitions and assumptions needed for mechanisms descriptions. The theme is perceived diversely.

Some, follow Jablonka (1995, 1998, 2005), associate this theme with Lamarck's approach as 'Lamarckian mechanisms'. This association needlessly and incorrectly suggests that Darwin's mechanism of natural selection is not needed to create adaptive changes. Emotions related to traditions of understanding of Lamarck make discussion difficult, so I try to relax them in (Gecow 2019a). However this approach discusses specific, real mechanisms, what brings them closer to explanation.

Others as in discussed Special Issue (2020) include that theme and mechanisms into term 'developmental bias'. It do not refer openly to the controversial ideas about Lamarck's views but question about mechanisms and source of adaptation in the term is hidden as a secondary, the first is identification and description of phenomena in the not precisely defined, provisory, wide range of the term. The term 'bias', however, also suggests additional source of adaptation than Darwinian natural selection (see ch.4.3), however, few articles in Special Issue openly promote such idea. It may leads to conclusion that Darwinian mechanism is not needed.

\subsection{Devbias4 is a result of natural selection}

Above (in ch.5.2) we conclude that the devbias4 is not a new source of the adaptations, it is only intermediary. In this point Laland with his co-authors do not agree, in their chapter " 4.3 Learning can generate "adaptation" without natural selection" (Laland et al. 2020) they write: "It is often claimed that natural selection is the only process that can systematically lead to increments in fitness across generations, but adaptive evolution can also result from phenotypic plasticity, habitat choice, and niche construction (Edelaar \& Bolnick, 2019).”. When we study current evolution (particular period of evolution), then devbias4 looks like independent (in considered

\footnotetext{
25 “developmental plasticity has the potential to exert developmental bias on variation in phenotype expression visible to selection" (Hu et al. 2020).

26 “...evolution exploits the underlying structural regularity of the environment to produce developmental systems that retain a bias toward phenotypes evolved in the past (Lipson et al. 2002; Watson et al. 2014). ” (Uller et al. 2018).

27 "Viewed from an engineering or design perspective, vertebrae number likely constrains the evolution of long, slender, and maneuverable necks in mammals." (Uller et al. 2018).

${ }^{28}$ https://extendedevolutionarysynthesis.com/ [March 30, 2020] Extended Evolutionary Synthesis, An integrative research program. Project leaders: Kevin Laland, Tobias Uller. The research project is funded by an grant from the John Templeton Foundation, supplemented by a further from host institutions. See also (Laland et al. 2015).
} 
period) source of adaptations and we can use it as such, but always should be remember, that earlier it was created by Darwinian mechanism (see also end of ch.4.3 and step 1 - creation of plasticity discussed in (Gecow 2019a)). I understand, that it is not always taken from observation, but Ockham's razor let us take such view, which we may add. Even learning while create new useful information, is a typical Darwinian mechanism: try and test then memorise useful result, later it is inheritance of such memes. The difference is only in elimination mechanism new mechanism needs not to kill, but it emerged by natural selection. Sources of plasticity and other devbiases4 I discuss in (Gecow 2019a).

Generally, term 'developmental bias' cover especially exciting theme: Are there other mechanisms creating adaptations than Darwinian natural selection? This is problem4. In (Parsons et al. 2020) can be found: "Specifically, Darwin's idea that variation is generated randomly has largely been taken for granted rather than tested, representing a fundamental gap in our understanding of evolution. ... the random generation of phenotypic variation posited by Darwin (1859) may not apply to cases of plasticity or even standard mutations." This refers only to 'current evolution' forgetting earlier stages, but they take, that assumption "variation is blind on needs" is necessary for Darwinian mechanism. It is wrong believing - changes have not to be blind, but they can (what is philosophically important) and this is enough to create adaptation by automatic eliminating those tries that are unable to survive (see ch.6.3).

\subsection{Limitation while information growth is not a developmental constraint}

Providing the result of the dice roll is an information - a limitation of the available selection. Similarly, when we learn that the distribution in phenotype space is not flat, but rather specific, we increase our knowledge (see ch.4.2). If the accepted change reduces the available areas of phenotype space, then it is an increase in the information held by the evolving object. Such change may indicate of better (in the sense of average fitness) phenotypes set. This is also indication of smaller set of useful areas. In my 'Draft of the deductive theory of life' (Gecow 2008, 2010) I call such information 'purposeful information'. Purposeful information cannot come from nowhere, natural (in wide meaning) selection is it's the only creator. May be, it is the way to prove, that there is no another mechanism of creation of adaptation.

The observed changes do not have to be a simple restriction (it is not a developmental constraints). They usually move the available area in the phenotype space to a new place. Describing the phenomenon in terms of purposeful information, it is not surprising that environmental correlations found on the occasion of 'current changes' (see ch.5.2), accepted by natural selection, can be also applied to next changes. In other words, the elements of a developmental mechanism that take into account these correlations while create change- 1 are used to canalization of change-2, although this change- 2 was caused by some new circumstances that are not usually a change of these correlations. The use of information is always a limitation. Considered above learning of environmental correlations is explained in (Uller et al. 2018) by "properties of regulatory networks evolving under natural selection ... As a result, evolving systems can exhibit bias toward phenotypes that are fit even in environments that have not been previously encountered, ...".

\subsection{Simple, abstract example for devbias4, randomness and blindness}

The term 'randomness' is used in several different meanings, which leads to confusion ${ }^{29}$. Changes in the Darwinian mechanism are 'random' which means - they are blind for needs ${ }^{\mathbf{3 0}}$ (Darwinian meaning of randomness), it does not mean that their probability distribution in fitness or phenotypic space (real meaning of randomness) is known and is isotropic (flat) (subjective meaning of randomness, see ch.4.2). It is also difficult to understand that the changes offered by devbias4 are still random and 'blind on needs' when in the definition of devbias4 it is openly stated that this distribution is more beneficial for the evolving object. The fact that the distribution is more beneficial does not necessarily mean that it more often generate acceptable changes or average fitness are higher. Other parameters of the distribution may be improved - acceptable changes occurring at the same frequency may give higher fitness, even average fitness may decrease in such a case, and the distribution may give significantly better results in the long run and as such should be included into devbias4. For simplicity, we will only consider the average fitness of the distribution (in the range of considered parameters).

These three variants of randomness are for many difficult to distinguish, to facilitate this distinction and give the basis for intuition let us follow the simple model of the evolving object. Considering devbias3, we pay special

29 "Neo-Darwinism did not come up with a definitive concept of blind, or 'random', variation. Authors of the Modern Synthesis, for instance, did not define the words 'random' or 'chance' mutation, and used these terms in a variety of ways, fostering misunderstanding of their theses [Merlin, 2010, 3]. ... Random mutations are not mutations that are all equally probable, or inherently unpredictable, or even causally independent from the environment [Sniegowski and Lenski, 1995, 572]. Nor does randomness mean that mutations will be equally likely to turn out beneficial, deleterious or neutral." (Pocheville, Danchin 2016).

30 "A core tenet of neo-Darwinism is that of blind variation, according to which heritable variations that fuels natural selection do not arise because of their adaptive value." (Pocheville, Danchin 2016). 
attention to changes in the probability distribution of random creation of a specific phenotypic change. In the model, some changes must therefore change the distribution. Probably, most hereditary developmental changes change the distribution of phenotypic changes in the next generation, and genes, or genes at the beginning (genetic assimilation), may not necessarily be the channel of hereditary information. What does it mean to draw a change that has been drawn recently? Usually other possible changes appear in the new situation. This distribution is undoubtedly an object feature subject to variability and selection. Until recently, it was thought that the distribution of genetic changes does not depend on the properties of the object, it is undoubtedly more difficult to control it, but phenotypic changes are already dependent on the genetic connections forming the complex network. In the model, however, we clearly distinguish between changes of distribution and 'current changes', which will not change the distribution. This will simplify considerations. Two variation distributions for 'current changes' will be used - one with equal probability of each change, corresponding to the assumption of isotropic variation (or 'flat'), which we will call 'subjective' (see ch.4.2) for convenience, and another distribution with different probability of changes, which we will call 'real'. The distribution changes will be carried out by changing the state of the drawing machine, and these changes belong to the set of randomized changes.

Table 1 shows both versions of the model. It has 4 rows distinguished by the letters $a, b, c, d$ for which mnemonic suggestions are given for the convenience of remembering. There are 16 possible changes, each is assigned a different number to distinguish them, which is given in the scope of the 'symbol of change'. There are 4 changes in each row. In the 'subjective' option a number from the 1-16 range is randomly drawn indicating the drawn change. In the 'real' option, a number from the 1-4 range is drawn indicating the row (the rows have the same probability and within the drawn row the change is drawn with the probability given in $\%$ in the 'probability of change in row [\%]' columns. In the 'effect of change: fitness or state' for the rows a, b, c is given change of fitness effected by this change in phenotype, and row $d-$ state switching of randomizing machine, wherein + and - indicate activation and deactivation of row 'a' or ' $b$ ' which defines the next machine state depending on the current state. Activation of the active row and deactivation of the inactive one is an empty operation, such as drawing a change from an inactive row. The machine has 4 states: AB, A, B, C. The rows ' $c$ ' and 'd' are always active (in any state). In the states $\mathrm{AB}, \mathrm{A}, \mathrm{B}$ a presence of letter indicates which row is active. So in state $\mathrm{AB}$ all lines are active, in $\mathrm{A}$ - the line ' $a$ ' is active, but ' $b$ ' is inactive, in $\mathrm{B}$ the opposite is true, in $\mathrm{C}$ - the lines ' $a$ ' and ' $b$ ' are inactive.

Both distributions - subjective and real give really random changes, according to the assumed distribution. The name 'subjective' refers to the reason for adopting a given distribution - it is estimated theoretically on the basis of premises, and 'real' is the result of measurement. The method of determining the distribution has no effect on the operation of the drawing machines, it is an element of model interpretation (ch.4.2). In the model, the 'real' distribution has been chosen so that each row gives the same 'mean fitness of row' as the 'subjective' distribution, where each change has the same probability of occurrence, making Table 2 common.

For rows a, b, c 'mean fitness of row' is defined and easy to calculate, while for row d the change in fitness depends on the state in which it is performed, as shown in Table 2, the probability of switching should be taken into account, which is not considered here anymore.

Considering that usually a decrease in fitness is not passed through natural selection, it is possible to evolve from state $\mathrm{B}$ to states $\mathrm{AB}(+\mathrm{a} 13)$ or to $\mathrm{C}$ (-b 16), and further to A (-b 16 or $+\mathrm{a} 13)$, but not between states $\mathrm{AB}$ and $\mathrm{C}$, despite the fact that they have the same average fitness, because it requires two changes, and one of them reduces fitness. Lack change of state is possible in this model only if a change from an active row other than ' $d$ ' is drawn. Acceptable changes of state in one step only give you an increase in average fitness by 1 . Changes in state requiring 2 steps are marked in italics.

Distribution changes are normal developmental changes, but in this article we call them 'devbiases3' (including devbiases4) because they are the result of comparing two distributions - earlier and later one. Each of these devbiases 3 has a specific attribute '+' (together with a neutral change, i.e. 0 in the last 4 columns of Table 2.) or ''. There is no value '@' for this attribute in this model, because there is no time needed for making the assessment by selection.

Table 1. Description in the text.

\begin{tabular}{|c|c|c|c|c|c|c|c|c|c|c|c|c|c|c|}
\hline \multicolumn{2}{|l|}{ row } & \multicolumn{4}{|c|}{ symbol of change } & \multicolumn{4}{|c|}{$\begin{array}{l}\text { effect of change: } \\
\text { fitness or state }\end{array}$} & \multirow{2}{*}{$\begin{array}{l}\begin{array}{l}\text { mean } \\
\text { fitness } \\
\text { of row }\end{array} \\
1\end{array}$} & \multicolumn{4}{|c|}{$\begin{array}{l}\text { probability of } \\
\text { change in row [\%] }\end{array}$} \\
\hline advance & a & 1 & 2 & 3 & 4 & 6 & 2 & -4 & 0 & & 10 & 40 & 10 & 40 \\
\hline bad & b & 5 & 6 & 7 & 8 & -7 & -9 & 5 & -1 & -3 & 13 & 40 & 33 & 14 \\
\hline constant & c & 9 & 10 & 11 & 12 & -1 & -7 & 1 & 3 & -1 & 9 & 32 & 22 & 37 \\
\hline distribution & $\mathrm{d}$ & 13 & 14 & 15 & 16 & $+\mathrm{a}$ & $-\mathrm{a}$ & $+\mathrm{b}$ & $-b$ & table 2 & 21 & 24 & 26 & 29 \\
\hline
\end{tabular}


Table 2. Description in the text.

\begin{tabular}{|r|c|c|c|c|c|c|}
\hline \multirow{2}{*}{$\begin{array}{l}\text { working } \\
\text { rows }\end{array}$} & \multirow{2}{*}{$\begin{array}{c}\text { mean } \\
\text { fitness } \\
\text { of state }\end{array}$} & \multicolumn{4}{|c|}{$\begin{array}{c}\text { mean fitness of change } \\
\text { from this state to new one }\end{array}$} \\
\cline { 5 - 8 } & from: AB & A & B & C \\
\hline a,b,c,d & AB & -1 & 0 & -1 & +1 & 0 \\
\hline a, c,d & A & 0 & +1 & 0 & +2 & +1 \\
\hline b,c,d & B & -2 & -1 & -2 & 0 & -1 \\
\hline c,d & C & -1 & 0 & -1 & +1 & 0 \\
\hline
\end{tabular}

We operate in the model on probability distributions. It is obvious that changes do not have to be random in their nature, but their causes and the changes themselves are taken from large and very diverse sets, and such choices are well described in statistics. The basic meaning of randomness is random taking according to such a distribution in opposition to the intentional choice, not based on the distribution, but on any intentions and knowledge of the choosing being. Differences between random changes and those leading to GMOs are a good example. In the model, we see how natural selection evolves from state B to state A.

Natural selection along with random variation is exactly 'playing 20 questions $^{31}$ - after each change the evolving object gets the answer 'YES' or 'NO'. These answers will increase its 'knowledge'. In my view (Gecow $2008,2010)$ this is 'purposeful information'. "Knowledge" is stored in the form of mechanisms in the object, and in the case of "knowledge about variability" - these mechanisms are devbias4. The drawing automaton in the model obtained such 'knowledge' (purposeful information) by finding the state A as better than B and AB or C. By comparing the states $\mathrm{B}$ and $\mathrm{AB}$ we know that there is devbias4 here - the average fitness of random changes increased significantly. In the state $\mathrm{AB}$, relative to the initial state $\mathrm{B}$, the changes are deliberately matched to the needs based on the acquired 'knowledge', but this is the result of a random change (+ a 13) that natural selection has approved. So object already has a part of the 'knowledge', but there remains an area that the 'object knows nothing' about and must draw there. The current distribution in the AB state concerns this area of volatility and in this range new changes are generated. These changes are completely random and are not selected based on the information: what fitness will be after it is generated. So they are 'blind on needs'. The only difference between 'current' (a, b, c) and 'distribution' (d) changes is the share of short-term and long-term effects in the aspect of fitness, which in this model were taken in extreme values and in the long-term limited to the distribution of next changes.

Attempts to formulate the experimental 'blindness'32 criterion quoted in (Pocheville, Danchin 2016) concern direction (presence of devbias), but not the 'blindness'. They assume that 'blindness' roughly means that the average fitness is 0 . Assumptions of this type are quite unsubstantiated and even erroneous (e.g. due to the expected degeneration, ch.2.2), and putting such a problem shows a misunderstanding of the essence of this wording.

What is the difference between blindness and randomness among themselves? Randomness is a statement of change generation compliance with (unknown or known, differentiated or flat) variability probability distribution. Whereas blindness is an element of explanation of where adaptation comes from, it is a statement that changes do not have to be intentional, i.e. they do not have to be selected to occur with some preference according to their fitness value in order for the natural selection mechanism to create adaptations. The picker may be blind to the resulting fitness value and may not be able to see and take it into account, but adaptation will increase. Therefore, intentionality is unnecessary and is not considered, then in the aspect of fitness the changes occur 'randomly', i.e. the fitness value of such changes is indefinite at the time the change occurs, and then assumes some 'random' value (i.e. unknown at the time of determining the change). In this sense, blindness is associated with randomness. The change would not be blind to the needs if, contrary to the currently working drawing mechanism and the resulting distribution, it obtained preferences (or the opposite) when it occurred depending on the needs. The needs of the

\footnotetext{
${ }^{31}$ The task is to guess the object by asking up to 20 questions, to which the answers can only be "yes" or "no".

32 "Merlin [2010] provides a useful review of several concept of blind variation. They can be classified according to whether they emphasize a statistical or a causal independence criterion between mutation and fitness (Table 1). The intuitive idea grounding these criteria is that for variation to be blind, we don't want more beneficial variations to be more probable in a given environment or, if variation shows such a pattern in a given environment, at least we don't want this pattern to be consistently conserved across different environments when the selective challenge changes." (Pocheville, Danchin 2016). This article captures blindness and its role in Darwinian selection very correctly and deeply, but it does not get to the bottom of the problem of the cause of misunderstanding. Blindness is different from not biasing, and randomness is another, third concept. These three concepts must be distinguished. The table there (in Merlin's version, Table 1, with slight modifications) is not for blindness, but for directed mutation.
} 
evolving object boil down to greater fitness, but the needs of the being generating change can be any, e.g. when creating GMOs.

So the model shows what devbias4 is and the scope of its fitness increase, as well as the range of randomness and blindness. Both ranges are separable (although the border is difficult to simply describe even in such a simple model), which eliminates the contradiction between the presence of devbias 4 and blindness. However, randomness is related to the distribution and it can be seen that it is not important whether the distribution is flat or differentiated. The change of the state of the object is here a normal random change and controlled in evolution by natural selection. Separating the collected devbiases4 from subsequent state changes is difficult, but the result of comparing subsequent states - a specific devbias4 is already clearly defined. From this it can be seen that problem 2 and devbias 2 were a lost project.

\section{Simplified assumptions of the view presented in (Uller et al. 2020)}

The article (Uller et al. 2020) indicates probably the most exactly the idea of 'developmental bias' therefore it needs special, more detailed analyse. Abstract contains: "Developmental plasticity looks like a promising bridge between ecological and developmental perspectives on evolution. Yet, there is no consensus on whether plasticity is part of the explanation for adaptive evolution or an optional "add-on" to genes and natural selection. Here, we suggest that these differences in opinion are caused by differences in the simplifying assumptions, and particular idealizations, that enable evolutionary explanation."

I agree, "that these differences in opinion are caused by differences in the simplifying assumptions", therefore the first step should be to indicate these simplifications.

Assumptions used in (Uller et al. 2020) article differ from e.g. my own what I indicate below. Primarily it is: 'Only current evolution is considered', means - only currently existing and active circumstances are taken as causes for considered evolutionary changes. Therefore 'natural selection' is limited to this considered period and plasticity looks like element independent on 'natural selection'. However we know, that 'natural selection' has worked also before considered period and plasticity is a result of it, than such independency is relative, but this is not noted, what makes false suggestion.

The "ecological perspective" is renamed in the text to "selective explanations" which shows it more openly to what the promoted idea "developmental perspective" as "transformational explanations" should be an alternative to. Explanation based on natural selection gives us clear mechanism of adaptation emergence, but alternative approach based on plasticity uses ready mechanisms that introduce adaptive changes. Here a tacit simplifying assumption is made that the causes (plasticity) of developmental changes are independent on the selective explanation, what makes symmetry of both approaches. For this symmetry a limitation to 'current evolution' is needed. However, deeply in the text can be found, that plasticity may be created thru natural selection.

"Looking for explanations based on fitness differences ('selective explanations'; Lewontin, 1983; Sober, 1984), makes it desirable to screen off other influences on evolution, even when these are potentially significant. ... any sustained directionality in evolution can be explained by referring to fitness differences between reliably inherited variants rather than to the introduction of variants." (Uller et al. 2020).

Then an ability of 'developmental plasticity' to introduction of variants that are hereditable and adaptive already while introduced, therefore they do not need selection, is the main newer than 'selective explanation' idea. Suggestion of symmetry of both approaches is the suggestion of their similar explanatory value but in particular cases one of them may be simpler and more useful. "The evolutionary process is incredibly complex, and biologists must choose which of its components to study." Therefore, although "any sustained directionality in evolution can be explained by referring to fitness" and Ockham razor can be used, we should look for alternative explanations.

However, is the explanation through developmental plasticity a real, symmetrical alternative? Out of range of 'current evolution' the plasticity was created by 'natural selection' (which is not questioned in (Uller et al. 2020)) and now it is only an intermediary but not an independent or another source of adaptation.

To take under consideration the (earlier collected) mechanisms proposing adaptive changes more frequently, than (at the beginning) blind on needs is the main goal of introducing the term 'developmental bias'. The bold text in brackets is added by me as especially important but omitted in (Uller et al. 2020). These collected mechanisms are there called 'plasticity', but currently it is too broad term. In (Gecow 2019a) I follow Jablonka call main part of it 'Lamarckian mechanisms', which include plasticity as prepared developmental reaction on environment stimuli, developmental regulation as prepared developmental reaction on deviation of development from the norm, and tuning of draw mechanism giving 'better' distribution. This last form of 'Lamarckian mechanisms' is the most interesting for the aim of 'devbias4'. It gives hereditable propositions. However, plasticity (in narrow sense) also fast gives phenotypic change needed to survive but it is typically not hereditable. Such change is then genetically assimilated (see Pocheville, Danchin 2016 or Gissis, Jablonka 2011) in such prepared and 'known' direction, but this needs more time although it is easier than without such a hint, and may 
be done by few steps (one complex step is not needed and much less probable). It makes important change in old 'selective explanation' to 'development first' approach. Regulations make a change 'smaller' therefore easier to accept by natural selection. These three type of mechanisms are different and should be discussed separately. Also form of plasticity used by (Jackson 2020) to describe ${ }^{33}$ PLE and CGV is a separate mechanism.

Summarizing, the term 'developmental bias' is to cover 'devbias4', which offer generation of heritable changes that can be: not blind on needs and be a source of adaptation independent on natural selection. I can agree for 'independent' in the period of current evolution, but not in general, as it is suggested, because devbias4 (which contains plasticity) was generated earlier by natural selection and during the period of current evolution it is only an intermediary. Used terminology, as the 'simplified assumption', is too wide, ambiguous and focused on collecting data but not on explaining it. The terminology needs deep analysis. In current form it has to lead to confusion and to a step back - i.e., to withdraw from the already attained awareness that there is no other source of adaptation than the Darwinian mechanism. Of course, we can look for other sources, but in this article, as in the whole Special Issue, it is suggested that such potential sources are already visible, which can hardly be considered grounded.

\section{Summary}

\subsection{System ordering the meaning of the term developmental bias}

The term 'development bias' promoted in the Special Issue is too broad, it mainly suggests other meanings than the creator of this slogan attributed to it. This leads to misunderstandings that must be clarified as soon as possible so that they do not hinder explanation and are not used outside of biology to depress evolution. The lack of definition of the term forced the search for the meaning also directly from the meaning of words 'developmental' and 'bias'. I propose to replace the lengthy two-word term with a shorter 'devbias' and use additional code on the end to indicate particular type of it. It will stabilize the position of the term in the Special Issue intention, but force more precision. I propose these codes, but it is not a closed system, it need deep and wider discussion.

There are 5 main types of devbiases denoted by digit:

- devbias1 - based on Arthur (2004) approach, defined theoretically using simple models, it is influence on direction (in fitness space) of evolution by factor present before selection and independent on current fitness landscape.

- devbias 2 - attempting to compare theoretically expected distribution of phenotypic changes to experimental data. In (Uller et al. 2018) approach, if the distribution is 'isotropic', then there is no bias (follow Arthur).

- devbias3 - differences found by comparison of two experimental distributions of phenotypic changes in the same evolutionary line typically looking on differences in their fitness effect.

- devbias4 - special case of devbias3 where later distribution exhibits higher mean fitness (is more useful for specie).

- $\quad$ pseudo-devbias5 - regularities in distributions of phenotypic changes acceptance looking like devbias3, also present before new selection, but distribution of phenotypic changes remain unchanged.

Presence (in distribution of phenotypic changes) before (' $b$ ') selection of a factor creating bias is the basic element of devbias phenomenon. However, it is typically possible to see only theoretically ('t'). Indicating such factor is a goal - explanation, but experiment (' $\mathrm{e}$ ') including observation can give us typically only distributions after (' $\mathbf{a}$ ') selection (or more exact in: small (' $\mathbf{s}$ '); middle (' $\mathbf{m}$ ') or great (' $\mathbf{g}$ ') selection period instead ' $\mathbf{a}$ '). Therefore e.g. devbias3ea is only a premise and hypothesis, that corresponding devbias 3 tb exists. In the exceptional case where the first appearance of change effects occurs at a sufficiently late stage of development, measurement before selection is possible.

The devbias3 has also important aspect of fitness effect: it may be '+' that means higher mean fitness (in the range of considered parameters) than in earlier distribution and such devbias3+ is a devbias4. It may be '-' that means lower mean fitness, but typically such the case is eliminated. And new distribution may be not assessed yet - it is at '@' selection in the time of observation due to long time needed for such assessing.

\footnotetext{
33 "Given that this cryptic [genetic (CGV)] variation may be sensitive to environmental triggers, it acts as a source of innovation that can be episodically induced and presented to selection (ref.). It is this form of plasticity, not adaptive plasticity, that plays the central role of driving the process of PLE [plasticity-led evolution] ... PLE is not an evolved response but rather the periodical accumulation and plastic release of CGV" (Jackson 2020).
} 
The devbias4 is the most exciting as a factor creating adaptation that is 'independent' on Darwinian natural selection. It can be treated as 'independent' only in the period of 'current evolution', but it is necessary to memorised, that it was earlier created by Darwinian natural selection and in the considered period it is only gobetween, intermediary mechanism. It is not a new source of the adaptations, that is truly independent on Darwinian mechanism.

Devbias4 is a mechanism adopted by natural selection that proposes new changes that are already adaptive. Such mechanisms were earlier called 'Lamarckian mechanisms'. In most cases they are plasticity (in old, narrow sense), however in both cases it is assumed, that such changes are heritable. Typically plastic changes are not heritable, they are only an adaptive reaction on environmental stimuli, but they may be supplemented by e.g. genetic assimilation.

The devbias1 was defined by Arthur (2004). This case should be denoted as devbias1btA. It indicates correlations of phenotypic change parameters making anisotropic distribution as a factor influencing direction of evolution. Due to this correlation present before selection the evolution goes to the top of fitness usually not by the shortest path - it has 'prejudice', i.e. bias. It may skip the nearest peak and reach a next one.

I have proposed devbias1btD that indicates degeneration as similar factor, but based on one parameter feature. For this case Wilkins (2020) shows 'domestication syndrome' that is probably devbias1geD.

The devbias 2 is in most cases subjective - it is too simple attempting to find Arthur's devbiases in observations. It is important to understand why it is too simple. For devbias 2 attribute ' $t / \mathrm{e}$ ' does not make sense.

Isotropic distribution is considered to be completely random, so bias is a deviation from it. This meaning was introduced by Arthur (2004) but bold in (Uller et al. 2018). It should be emphasized here that the randomness of variation in the Darwinian theory of evolution does not mean, however, isotropic variability, but that variability can be (it has not to be) blind for the needs of adaptation. The changes are random, but their probability distribution is not indicated, in particular it does not have to be isotropic distribution. In anisotropic distribution some directions are preferred, but they are random (until they are verified by selection). The deviation from flat randomness is a subjective feature, because there is no deviation from the objectively existing distribution, but from the alleged distribution assuming 'provisionally' the absence of differentiating factors. However, objective correlation objectively drives evolution.

Devbiases 4 are those of devbiases3 that selection stated that they increase (at least do not reduce) probability of acceptance (mean fitness) of the newly generated changes, comparing to the distribution before creating devbias4. Then this new distribution is 'biased', giving more desirable results than the previous one, which was not suspected of bias, at least in this new aspect, i.e. we considered it to be 'fairly' random. Devbias4 is the case that from the beginning creates the intuitive meaning of devbias in general, i.e. also includes devbias1 and devbias3, but we must be aware ${ }^{34}$ that this intuition only applies to devbias4.

Until the Darwinian natural selection decides on the mechanism of a given devbias3@ (including later devbias4), it has the status of a new trait being tested, it can harm or help at that time. After this transitional period, some devbias3@ acquires the rights ('+') of a tested mechanism adopted by ordinary natural selection, which thus becomes a source of increased adaptability of the new traits proposed through this devbias4. However, this is comparison to the earlier distribution which ceased working. But the currently working (new and 'better') distribution still produces fully random and blind for the needs changes.

\subsection{Conclusion}

Devbias4 generates variability, which can be described as 'non-random' (comparing to the situation without this devbias4) and even 'resulting from the instruction' which is the devbias4 mechanism. The presence of this mechanism affects the direction of evolution, but the variability is still random, according to the current probability distribution which has been modified by natural selection. Under this current distribution, variability is blind for the needs, it ceases to be a blind under the previous, already outdated and non-operative distribution. Darwinian natural selection, which had randomly generated and left devbias 4 as a result of the test, remains the only source of adaptability (resulting from devbias4) of new changes. So devbias4 is only an intermediary, not an independent source of adaptation.

Considering the current evolution, we really have two separate sources of adaptation - one resulting from accumulated devbias4 (representing the previous operation of selection on the distribution), and the remain is the

\footnotetext{
${ }^{34}$ As example see two first statement in Introduction to (Jackson 2020): "Developmental bias describes the manner in which certain changes to development are more accessible to evolution than others (Arthur, 2004). A key generator of developmental bias, and integral to understanding its role in evolution, is developmental plasticity, which refers to an organism's ability to adjust its phenotype in response to environmental conditions (Laland et al., 2015; Schwab, Casasa, \& Moczek, 2019).”
} 
work of the current natural selection of traits currently drawn randomly according to the current probability distribution.

The wording "Phenotypic variation is generated by the processes of development, with some variants arising more readily than others - a phenomenon known as "developmental bias". "(Uller et al. 2018) suggests that considering current evolution we should assess the randomness of variability not according to the actual real distribution, but in relation to some primary, imaginary, abstract distribution that does not take into account the achievements of selection in the form of accumulated devbias4. As a result of such a methodologically wrong approach, actually there are noted "biases" that give adaptations from a sources that are not yet defined. These sources are clearly "other than the Darwinian selection" which is questioned ${ }^{35}$. Such a clear conclusion from the scientific approach can easily penetrate non-scientific recipients. Some societies (easy to pointing them out) can count on it. Deeper, complex considerations on the emergence of those various sources of adaptation unreasonably and incorrectly called 'non-Darwinian' are already 'enough' illegible for those 'non-scientific' recipients. This creates a field for their own assessments based on traditional concepts.

The promotion of the term 'developmental bias' clearly leads in the opposite direction to the desirable explanation of the real sources of adaptation. This topic is particularly important in social space, what requires special responsibility of scientists. Instead of explaining, this term confuses. It is too broad, very poorly defined (which is emphasized by many authors of Special Issue cited earlier) and incorrectly suggests the presence of objective biases already in the range of devbias2. It creates a conceptual structure that is not useful for explanation, but useful for non-scientific undermining the greatest achievements of evolutionary biology. The principles of creating this structure are consistent with the tradition in biology of paying attention to an in-depth description of phenomena, while leaving the explanation into the next, clearly delimited stage, which is approached with caution limiting 'speculation' to a minimum. At present, this tradition significantly hinders explanation and should be overcome. The first step should be choosing well-defined terms for specific mechanisms ${ }^{36}$, and departing from overly general descriptive / phenomenological terms, as the postulated term 'developmental bias' is.

\section{References}

Altenberg L. 2005 Modularity in Evolution: Some Low-Level Questions. In: Modularity : understanding the development and evolution of natural complex systems. W. Callebaut and D. Rasskin-Gutman (eds), The Vienna series in theoretical biology, MIT Press, 99-128.

Arthur W. 2004 The effect of development on the direction of evolution: toward a twenty-first century consensus. Evolution and Development. 6 (4): 282-288. doi:10.1111/j.1525-142x.2004.04033.x, ISSN 1520-541X, PMID 15230968.

de Beer G. 1940 Embryos and Ancestors. Oxford f.e.

Brigandt I. 2020 Historical and philosophical perspectives on the study of developmental bias. Evolution \& Development. 22:7-19. https://doi.org/10.1111/ede.12302

Draghi J. 2020 Developmental Noise and Ecological Opportunity Across Space Can Release Constraints on the Evolution of Plasticity. Evolution \& Development. 22:35-46. https://doi.org/10.1111/ede.12305

Gecow A. 2005 From a "Fossil" Problem of Recapitulation Existence to Computer Simulation and Answer. Neural Network World. 3/2005, 189-201 Inst. Computer Sci. Acad. Sci. Czech Rep.

Gecow A. 2008 The purposeful information. On the difference between natural and artificial life. Dialogue \& Universalism $11-12,191-206$

Gecow A. 2009a Emergence of Growth and Structural Tendencies During Adaptive Evolution of System. In: From System Complexity to Emergent Properties. M.A. Aziz-Alaoui \& Cyrille Bertelle (eds), Springer, Understanding Complex Systems Series, 211-241.

Gecow A. 2009b The Simple Model of Living Object as an Outside State of Statistical Stable Equilibrium, the Small Change Tendency in Adaptive Evolution. In: Modelling and Analysis of Complex Interacting Systems. M.A. Aziz-Alaoui \& Cyrille Bertelle (eds), DCDIS-B special issue, 515-533.

Gecow A. 2010 The differences between natural and artificial life, towards a definition of life. http://arxiv.org/abs/1012.2889

Gecow A. 2019a Darwinian base of the Lamarckian mechanisms, Extended Synthesis as a step towards deductive theory (full version) https://osf.io/yr $2 \mathrm{~h} 9$

Gecow A. 2019b Life is not on the edge of chaos but in a half-chaos of not fully random systems. Definition and simulations of the half-chaos in complex networks, http://arxiv.org/abs/1712.09609 v3, currently accepted for publication in the book under the working title Chaotic Systems, P. Bracken (ed), IntechOpen, ISBN 978-1-83962-859-7.

Gissis SB, Jablonka E. (eds), 2011 Transformations of Lamarckism: From Subtle Fluids to Molecular Biology. The Vienna Series in Theoretical Biology, The MIT Press, Cambridge, Massachusetts, London, England.

Hordijk W, Altenberg L. 2020 Developmental structuring of phenotypic variation: A case study with a cellular automata model of ontogeny. Evolution \& Development. 22:20-34. https://doi.org/10.1111/ede.12315

\footnotetext{
35 (Uller et al. 2018): "That phenotypic variation is unbiased has ... probably been the default assumption in evolutionary theory. ... this assumption is likely to be unfounded."

${ }^{36}$ Jablonka has been doing this in the subject since it was revitalized. In the Special Issue she is completely omitted from references.
} 
Hu Y, Linz DM, Parker ES, Schwab DB, Casasa S, Macagno ALM, Moczek AP. 2020 Developmental bias in horned dung beetles and its contributions to innovation, adaptation, and resilience. Evolution \& Development. 22:165-180. https://doi.org/10.1111/ede.12310

Jablonka E, Lamb MJ. 1995 Epigenetic Inheritance and Evolution: The Lamarckian Dimension. Oxford: Oxford University Press.

Jablonka E, Lamb MJ, Avital E. 1998 Lamarckian' mechanisms in Darwinian evolution TREE 13. 206-210.

Jablonka E, Lamb MJ. 2005 Evolution in Four Dimensions: Genetic, Epigenetic, Behavioral, and Symbolic Variation in the History of Life. Cambridge, MA: MIT Press. (Revised edition at 2014.)

Jablonka E, Lamb MJ. 2007 The expanded evolutionary synthesis - a response to Godfrey-Smith, Haig, and West-Eberhard Biol Philos 22: 453-472

Jablonka E, Lamb MJ. 2008 Soft inheritance: Challenging the modern synthesis. Genetics and Molecular Biology 31 (2): 389-395.

Jablonski D. 2020 Developmental bias, macroevolution, and the fossil record. Evolution \& Development. 22:103-125. https://doi.org/10.1111/ede.12313

Jackson ISC. 2020 Developmental bias in the fossil record. Evolution \& Development. 22:88-102. https://doi.org/10.1111/ede.12312

Kavanagh K. 2020 Developmental plasticity associated with early structural integration and evolutionary patterns: Examples of developmental bias and developmental facilitation in the skeletal system. Evolution \& Development. 22:196-204. https://doi.org/10.1111/ede.12323

Laland K, Uller T, Feldman M, Sterelny K, Müller GB, Moczek A, Jablonka E, Odling-Smee J. 2014 Does evolutionary theory need a rethink? Yes, urgently. Nature, V514, 9

Laland KN, Uller T, Feldman MW, Sterelny K, Müller GB, Moczek A, Jablonka E, Odling-Smee J. 2015 The extended evolutionary synthesis: its structure, assumptions and predictions Proc. R. Soc. B 282: 20151019.

Laland KN, Toyokawa W, Oudman T. 2020 Animal learning as a source of developmental bias. Evolution \& Development. 22:126-142. https://doi.org/10.1111/ede.12311

Levis NA, Pfennig DW. 2020 Plasticity-led evolution: A survey of developmental mechanisms and empirical tests. Evolution \& Development. 22:71-87. https://doi.org/10.1111/ede.12309

Moczek A. (ed) 2020 Developmental Bias in Evolution Special Issue of 'Evolution \& Development' Volume22, Issue1-2 https://doi.org/10.1111/ede. 12330

Moczek A. 2020 Biases in the study of developmental bias. Evolution \& Development. 22: 3-6 DOI: 10.1111/ede.12325

Naef A. 1917 Die individuelle Entwicklung organischen Formen als Urkunde ihrer Stammesgeschichte. Jena.

Parsons KJ, McWhinnie K, Pilakouta N, Walker L. 2020 Does phenotypic plasticity initiate developmental bias? Evolution \& Development. 22:56-70. https://doi.org/10.1111/ede.12304

Pocheville A, Danchin É. 2016 Genetic assimilation and the paradox of blind variation. In book: Challenging the Modern Synthesis Editors: Philippe Huneman, Denis Walsh, Publisher: Oxford University Press. DOI: 10.1093/oso/9780199377176.003.0003

Uller T, Moczek AP, Watson RA, Brakefield PM, Laland KN. 2018 Developmental bias and evolution: A regulatory network perspective. Genetics 209:1-18 pp.949-966 DOI: 10.1534/genetics.118.300995.

Uller T, Feiner N, Radersma R, Jackson ISC, Rago A. 2020 Developmental plasticity and evolutionary explanations. Evolution \& Development. 22:47-55. https://doi.org/10.1111/ede.12314

West-Eberhard MJ. 2003 Developmental Plasticity and Evolution. New York: Oxford University Press.

Wilkins AS. 2020 A striking example of developmental bias in an evolutionary process: The "domestication syndrome". Evolution \& Development. 22:143-153. https://doi.org/10.1111/ede.12319 